\title{
Nonlinear diffusion equations driven by the $p(\cdot)$-Laplacian
}

\author{
Goro Akagi and Kei Matsuura
}

\begin{abstract}
This paper is concerned with nonlinear diffusion equations driven by the $p(\cdot)$-Laplacian with variable exponents in space. The wellposedness is first checked for measurable exponents by setting up a subdifferential approach. The main purposes are to investigate the large-time behavior of solutions as well as to reveal the limiting behavior of solutions as $p(\cdot)$ diverges to the infinity in the whole or in a subset of the domain. To this end, the recent developments in the studies of variable exponent Lebesgue and Sobolev spaces are exploited, and moreover, the spatial inhomogeneity of variable exponents $p(\cdot)$ is appropriately controlled to obtain each result.
\end{abstract}

Mathematics Subject Classification (2010). Primary 35K92; Secondary $35 \mathrm{~K} 55,46 \mathrm{E} 35$.

Keywords. $p(\cdot)$-Laplacian, Nonlinear diffusion, Subdifferential, Parabolic equation, Variable exponent Lebesgue, Sobolev spaces.

\section{Introduction}

Nonlinear elliptic operators with non-standard growth have been attracting more attention in the studies of nonlinear PDEs. The reader can overview the recent development of this field in [28]. Here we particularly treat the $p(\cdot)$-Laplace operator $\Delta_{p(\cdot)}$ given by

$$
\Delta_{p(\cdot)} \phi(x):=\nabla \cdot\left(|\nabla \phi(x)|^{p(x)-2} \nabla \phi(x)\right)
$$

with a measurable function $p(\cdot)$ from $\Omega \subset \mathbb{R}^{N}$ into $(1, \infty)$. The $p(\cdot)$-Laplacian with a variable exponent $p(\cdot)$ is deeply related to generalized Lebesgue and Sobolev spaces, $L^{p(\cdot)}$ and $W^{1, p(\cdot)}$, which have been vigorously studied and

Dedicated to Professor Tomomi Kojo on the occasion of his 60th birthday.

This work was supported in part by the Shibaura Institute of Technology grant for Project Research, and the Grant-in-Aid for Young Scientists (B) (No. 22740093), Ministry of Education, Culture, Sports, Science and Technology. 
whose theory has been ripe for applications to PDEs (see [20]). There have been many contributions to nonlinear elliptic problems associated with the $p(\cdot)$-Laplacian from various view points (see [28] for a survey). On the other hand, there seem to be less contributions to parabolic problems.

Let $\Omega \subset \mathbb{R}^{N}$ be a bounded domain with smooth boundary $\partial \Omega$. In this paper, we deal with a solution $u=u(x, t)$ for the following initial-boundary value problem:

$$
\begin{aligned}
\partial_{t} u=\Delta_{p(\cdot)} u+f & \text { in } \Omega \times(0, \infty), \\
u=0 & \text { on } \partial \Omega \times(0, \infty), \\
u(\cdot, 0)=u_{0} & \text { in } \Omega,
\end{aligned}
$$

where $\partial_{t} u=\partial u / \partial t$ and $f: \Omega \times(0, \infty) \rightarrow \mathbb{R}$ and $u_{0}: \Omega \rightarrow \mathbb{R}$ are given functions. Equation (1.1) can be regarded as a sort of nonlinear diffusion equation, whose diffusion coefficient is of the form $|\nabla u(x, t)|^{p(x)-2}$ by analogy with Fick's diffusion model. Hence the nonlinear diffusion driven by (1.1) strongly depends on the gradient of the density $u(x, t)$, and moreover, it might be inhomogeneous in space even though the gradient has the same norm over $\Omega$. The constant exponent $p(\cdot) \equiv p$ is particularly known to have a threshold between two drastically different types of nonlinear diffusion, and moreover, the limit of $p \rightarrow \infty$ exhibits a peculiar phenomena called fast/slow diffusion.

Parabolic equations involving the $p(\cdot)$-Laplacian have been proposed in the study of image restoration (see [17]) as well as in some model of electrorheological fluids (see $[19,21,34]$ ). A mathematical analysis was also done for such problems by Acerbi and Mingione [1,2] and by Acerbi et al. [3]. In [27], some nonlinear parabolic problem proposed by [17] was studied in a weak formulation and an existence result for weak solutions was established. Antontsev and Shmarev studied parabolic equations involving anisotropic $p(\cdot, \cdot)$-Laplace operators with log-Hölder continuous $(x, t)$-dependent exponents and proved the existence, uniqueness, extinction in finite time and blow-up of solutions in [7-10]. Equation (1.1) was also studied by Bendahmane et al. [15], where the well-posedness is proved for renormalized solutions in an $L^{1}$-framework for continuous variable exponents. Moreover, the existence and uniqueness of entropy solutions and the equivalence between two notions of solutions are discussed for log-Hölder continuous variable exponents in [38] by Zhang and Zhou (see also [36] for an elliptic counterpart).

The main purpose of this paper is to observe specific properties of solutions for the nonlinear diffusion equation (1.1)-(1.3) with a variable exponent $p(\cdot)$. To be concrete, we shall investigate asymptotic behaviors of solutions $u=u(x, t)$ as $t \rightarrow \infty$ when $f \equiv 0$, and we shall also find out the limiting behavior of solutions for $p_{n}(\cdot)$-Laplacians as $p_{n}(\cdot) \rightarrow \infty$. To do so, we work in an $L^{2}$-framework and set up a subdifferential approach to (1.1). In Sect. 2, we recall the definition of variable exponent Lebesgue spaces, $L^{p(\cdot)}(\Omega)$, as well as Sobolev spaces, $W^{1, p(\cdot)}(\Omega)$. Moreover, some properties of these spaces will be also exhibited to be used later. In Sect. 3, we discuss the well-posedness of the Cauchy-Dirichlet problem (1.1)-(1.3) with measurable exponents $p(\cdot)$ 
by using a standard theory of evolution equations governed by subdifferential operators. It is noteworthy that our well-posedness result is completely free from continuity assumptions on variable exponents. Indeed, it has been an open question whether the (log-Hölder) continuity of variable exponent is necessary for the well-posedness (see p. 4560 of [28]).

Section 4 is devoted to revealing asymptotic behaviors as $t \rightarrow \infty$ of solutions for (1.1)-(1.3) without source (i.e., $f \equiv 0)$. For the fully degenerate case, $\inf p(\cdot)>2$, solutions decay and converge to 0 as $t \rightarrow \infty$. On the other hand, for the fully singular case, $\sup p(\cdot)<2$, one can observe the extinction of solutions, namely, every solution vanishes at a finite time, which is called extinction time. We estimate the decay rate and the extinction rate of solutions from above and below. Moreover, we also establish an estimate for the extinction time in terms of initial data.

In contrast to constant exponent cases, where decay and extinction rates are homogeneous in space, variable exponents might turn them inhomogeneous. Moreover, when $p(x) \equiv p$, the non-increase in $t$ of the Rayleigh quotient $\|\nabla u(\cdot, t)\|_{L^{p}} /\|u(\cdot, t)\|_{L^{2}}$ plays a crucial role to obtain decay/extinction rates of solutions. However, in variable exponent cases, it would be somewhat difficult to obtain the non-increase of the corresponding Rayleigh quotient with $L^{p(\cdot)}(\Omega)$-norm, due to the lack of homogeneity of the modular in $L^{p(\cdot)}(\Omega)$-space.

In Sect. 5, we investigate the fast/slow diffusion limit, that is, the limit as $n \rightarrow \infty$ of solutions $u_{n}=u_{n}(x, t)$ for the nonlinear diffusion (1.1)-(1.3) with a sequence of variable exponents $p_{n}(\cdot) \rightarrow \infty$. The constant exponent version of fast/slow diffusion limit arises from some macroscopic model of a critical-state of type-II superconductors and a model of sandpile growth (see $[6,11,13])$. Moreover, the variable exponent case could be a natural extension to these physical models. Since the diffusion coefficient for the $p_{n}(\cdot)$-Laplacian is in the form $|\nabla u(x, t)|^{p_{n}(x)-2}$ with the density $u(x, t)$ at the position $x$ and the time $t$, one can expect that the speed of diffusion diverges as $p_{n}(x) \rightarrow \infty$ at $(x, t)$ where $|\nabla u(x, t)|$ is greater than 1 and no diffusion will occur at $(x, t)$ where $|\nabla u(x, t)|$ is less than 1. Such a limiting diffusion is called fast/slow diffusion in [11]. Compared to the constant exponent case, the speed of the divergence of $p_{n}(\cdot)$ might not be uniform over $\Omega$ in our setting. We prove the convergence of solutions as $n \rightarrow \infty$ and find out a limiting problem, which is an evolutionary variational inequality with a constraint set of the form,

$$
K=\left\{w \in H_{0}^{1}(\Omega) ;\|\nabla w\|_{L^{\infty}(\Omega)} \leq 1\right\},
$$

under an appropriate control of the fastest and slowest speeds of $p_{n}(\cdot) \rightarrow \infty$ over $\Omega$. Our analysis is based on a gradient structure of (1.1) and the notion of Mosco convergence for convex functionals is employed to investigate the limit of Lyapunov functionals.

In the final section, we treat a more peculiar case, where $p_{n}(\cdot)$ diverges only on a subset of $\Omega$. More precisely, we consider the case that 


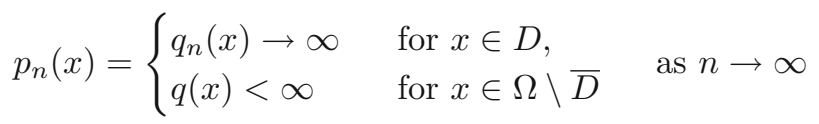

with an open subset $D$ of $\Omega$ and measurable functions $q_{n}(\cdot), q(\cdot)$ with values in $(1, \infty)$. From an analogue to the former case, one can expect that the fast/slow diffusion can be observed in $D$ by letting $n \rightarrow \infty$, and moreover, an inhomogeneous nonlinear diffusion driven by the $q(\cdot)$-Laplacian occurs in $\Omega \backslash \bar{D}$. This situation is particular to the variable exponent case and never happens in the constant exponent case. We finally obtain a mixed problem which consists of an evolutionary quasi-variational inequality in $D$ and a nonlinear diffusion equation involving the $q(\cdot)$-Laplacian in $\Omega \backslash \bar{D}$ as a limiting problem. In contrast to the preceding case, the constraint set of the quasi-variational inequality depends on the unknown.

We also have to mention an elliptic counterpart of the limiting problems treated here. In [31], Manfredi et al. studied the limits as $p_{n}(x) \rightarrow \infty$ of $p_{n}(x)$ harmonic functions, i.e., solutions of $-\Delta_{p_{n}(\cdot)} u_{n}=0$ in $\Omega$. They finally proved that the limit of $u_{n}$ solves an elliptic equation involving the so-called infinityLaplace operator under more restrictive assumptions of $p_{n}(x) \rightarrow \infty$ than ours (see also Remark 5.4 in Sect. 5). Moreover, in [30], they also treated the case that $p_{n}(x) \rightarrow \infty$ only in a subdomain of $\Omega$ (see also [37]). However, we emphasize that the elliptic limit would be essentially different from parabolic ones. In parabolic cases, due to the presence of the time-derivative term, one cannot normalize both sides of equations when $p_{n}(x)$ diverges to the infinity, and hence, the limiting problems are described as variational inequalities instead of PDEs involving explicit differential operators such as the infinity-Laplacian.

Notation. We write $(s)_{+}:=\max \{s, 0\}$ for $s \in \mathbb{R}$. Let $\|\cdot\|_{q}$ denote the usual norm of $L^{q}(\Omega)$-spaces for $1 \leq q \leq \infty$. Moreover, $(\cdot, \cdot)_{L^{2}}$ denotes the usual inner product of the Hilbert space $L^{2}(\Omega)$, i.e., $(u, v)_{L^{2}}=\int_{\Omega} u(x) v(x) d x$.

\section{Preliminaries}

This section is devoted to some preliminary results on Lebesgue and Sobolev spaces with variable exponents (see $[22,23,25,29]$ for a good introduction to this field and [20] for a complete collection of up-to-date results). Let $\Omega$ be a domain in $\mathbb{R}^{N}$. Throughout this section, we assume that $p$ is a measurable function from $\Omega$ to $[1, \infty)$. We write

$$
p^{+}:=\operatorname{ess} \sup _{x \in \Omega} p(x), \quad p^{-}:=\operatorname{ess} \inf _{x \in \Omega} p(x) .
$$

Define the Lebesgue space with a variable exponent $p(\cdot)$, which is the so-called Nakano space and a special sort of Musielak-Orlicz spaces (see [32]), as follows:

$$
L^{p(\cdot)}(\Omega):=\left\{u: \Omega \rightarrow \mathbb{R} ; \text { measurable in } \Omega \text { and } \int_{\Omega}|u(x)|^{p(x)} d x<\infty\right\}
$$


with a Luxemburg-type norm

$$
\|u\|_{p(\cdot)}:=\inf \left\{\lambda>0 ; \int_{\Omega}\left|\frac{u(x)}{\lambda}\right|^{p(x)} d x \leq 1\right\} .
$$

The following proposition plays an important role to establish energy estimates (see, e.g., Theorem 1.3 of [25] for a proof).

Proposition 2.1. It holds that

$$
\sigma^{-}\left(\|w\|_{p(\cdot)}\right) \leq \int_{\Omega}|w(x)|^{p(x)} d x \leq \sigma^{+}\left(\|w\|_{p(\cdot)}\right) \quad \text { for all } w \in L^{p(\cdot)}(\Omega)
$$

with the strictly increasing functions

$$
\sigma^{-}(s):=\min \left\{s^{p^{-}}, s^{p^{+}}\right\}, \quad \sigma^{+}(s):=\max \left\{s^{p^{-}}, s^{p^{+}}\right\} \quad \text { for } s \geq 0 .
$$

We next define variable exponent Sobolev spaces $W^{1, p(\cdot)}(\Omega)$ as follows:

$$
W^{1, p(\cdot)}(\Omega):=\left\{u \in L^{p(\cdot)}(\Omega) ; \frac{\partial u}{\partial x_{i}} \in L^{p(\cdot)}(\Omega) \quad \text { for all } i=1,2, \ldots, N\right\}
$$

with the norm

$$
\|u\|_{W^{1, p(\cdot)(\Omega)}}:=\left(\|u\|_{p(\cdot)}^{2}+\|\nabla u\|_{p(\cdot)}^{2}\right)^{1 / 2}
$$

where $\|\nabla u\|_{p(\cdot)}$ denotes the $L^{p(\cdot)}(\Omega)$-norm of $|\nabla u|$. Furthermore, let $W_{0}^{1, p(\cdot)}(\Omega)$ be the closure of $C_{0}^{\infty}(\Omega)$ in $W^{1, p(\cdot)}(\Omega)$. Here we note that the space $W_{0}^{1, p(\cdot)}(\Omega)$ is usually defined in a slightly different way for the variable exponent case. However, both definitions are equivalent under (2.1) given below (see [20] and also [39] for an unusual phenomenon of discontinuous exponents). In this paper, we use the notation of $W_{0}^{1, p(\cdot)}(\Omega)$ only when $(2.1)$ is satisfied.

The following proposition is concerned with the uniform convexity of $L^{p(\cdot)}$ - and $W^{1, p(\cdot)}$-spaces.

Proposition 2.2. ([20]) If $p^{-}>1$ and $p^{+}<\infty$, then $L^{p(\cdot)}(\Omega)$ and $W^{1, p(\cdot)}(\Omega)$ are uniformly convex Banach spaces. Hence they are reflexive.

Let us exhibit the Poincaré and Sobolev inequalities (see [24,26,35] and references therein for more details). To do so, we introduce the log-Hölder condition:

$$
\left|p(x)-p\left(x^{\prime}\right)\right| \leq \frac{A}{\log \left(e+1 /\left|x-x^{\prime}\right|\right)} \quad \text { for all } x, x^{\prime} \in \Omega
$$

with some constant $A>0$ (see [20]). This condition follows from the Hölder continuity of $p$ over $\bar{\Omega}$ and it implies $p \in C(\bar{\Omega})$ and $p^{+}<\infty$.

Proposition 2.3. ([20]) Let $\Omega$ be a bounded domain in $\mathbb{R}^{N}$ with smooth boundary $\partial \Omega$. Assume that (2.1) holds.

(i) There exists a constant $C \geq 0$ such that

$$
\|w\|_{p(\cdot)} \leq C\|\nabla w\|_{p(\cdot)} \quad \text { for all } w \in W_{0}^{1, p(\cdot)}(\Omega) .
$$


In particular, the space $W_{0}^{1, p(\cdot)}(\Omega)$ has a norm $\|\cdot\|_{1, p(\cdot)}$ given by

$$
\|w\|_{1, p(\cdot)}:=\|\nabla w\|_{p(\cdot)} \quad \text { for } \quad w \in W_{0}^{1, p(\cdot)}(\Omega),
$$

which is equivalent to $\|\cdot\|_{W^{1, p(\cdot)}(\Omega)}$.

(ii) Let $q: \Omega \rightarrow[1, \infty)$ be a measurable and bounded function and suppose that $q(x) \leq p^{*}(x):=N p(x) /(N-p(x))+$ for a.e. $x \in \Omega$. Then $W^{1, p(\cdot)}(\Omega)$ is continuously embedded in $L^{q(\cdot)}(\Omega)$.

In particular, if $\Omega$ is bounded and smooth, $p^{-} \geq 2 N /(N+2)$ and (2.1) holds, then there exists a constant $C_{p(\cdot), 2}>0$ such that

$$
\|w\|_{2} \leq C_{p(\cdot), 2}\|w\|_{1, p(\cdot)} \quad \text { for all } w \in W_{0}^{1, p(\cdot)}(\Omega) .
$$

This fact will be frequently used in Sect. 4 .

Let us introduce the following amalgam space with a variable exponent:

$$
X^{p(\cdot)}(\Omega):=\left\{u \in L^{2}(\Omega) ; \partial u / \partial x_{i} \in L^{p(\cdot)}(\Omega) \text { for } i=1,2, \ldots, N\right\}
$$

equipped with the norm

$$
\|u\|_{X^{p(\cdot)}(\Omega)}:=\left(\|u\|_{2}^{2}+\|\nabla u\|_{p(\cdot)}^{2}\right)^{1 / 2} \quad \text { for } u \in X^{p(\cdot)}(\Omega) .
$$

Moreover, set a subspace of $X^{p(\cdot)}(\Omega)$ by

$$
X_{0}^{p(\cdot)}(\Omega):=X^{p(\cdot)}(\Omega) \cap W_{0}^{1, p^{-}}
$$

with $\|u\|_{X_{0}^{p(\cdot)}(\Omega)}:=\|u\|_{X^{p(\cdot)}(\Omega)}$. Assume that $1<p^{-}$and $p^{+}<\infty$. Then since $L^{2}(\Omega)$ and $L^{p(\cdot)}(\Omega)$ are reflexive Banach spaces, one can observe that $X^{p(\cdot)}$ is a reflexive Banach space, and $X_{0}^{p(\cdot)}(\Omega)$ is as well, since $X_{0}^{p(\cdot)}(\Omega)$ is a closed subspace of $X^{p(\cdot)}(\Omega)$.

If $\Omega$ is bounded and smooth, then it follows that

$$
W^{1, p^{+}}(\Omega) \cap L^{2}(\Omega) \hookrightarrow X^{p(\cdot)}(\Omega) \hookrightarrow W^{1, p^{-}}(\Omega) \cap L^{2}(\Omega)
$$

with continuous canonical injections. Indeed, $X^{p^{+}}(\Omega) \hookrightarrow X^{p(\cdot)}(\Omega) \hookrightarrow X^{p^{-}}(\Omega)$ continuously by $p^{-} \leq p(\cdot) \leq p^{+}$, and moreover, $X^{p^{-}}(\Omega) \hookrightarrow W^{1, p^{-}}(\Omega)$ continuously by usual Sobolev's embedding theorem. Since $W^{1, p^{+}}(\Omega) \cap L^{2}(\Omega) \hookrightarrow$ $X^{p^{+}}(\Omega)$ clearly, we obtain (2.3). It also follows that

$$
W_{0}^{1, p^{+}}(\Omega) \cap L^{2}(\Omega) \hookrightarrow X_{0}^{p(\cdot)}(\Omega) \hookrightarrow W_{0}^{1, p^{-}}(\Omega) \cap L^{2}(\Omega) .
$$

Furthermore, we have:

Proposition 2.4. Assume that $\Omega$ is bounded and smooth. If $p(\cdot)$ satisfies (2.1), then $X^{p(\cdot)}(\Omega)$ and $X_{0}^{p(\cdot)}(\Omega)$ coincide with $W^{1, p(\cdot)}(\Omega) \cap L^{2}(\Omega)$ and $W_{0}^{1, p(\cdot)}(\Omega) \cap$ $L^{2}(\Omega)$, respectively.

Proof. By Corollary 8.2.6 of [20], there exists a constant $C$ such that

$$
\left\|u-\langle u\rangle_{\Omega}\right\|_{p(\cdot)} \leq C\|\nabla u\|_{p(\cdot)} \quad \text { for } u \in L_{l o c}^{1}(\Omega) \text { satisfying }|\nabla u| \in L^{p(\cdot)}(\Omega),
$$

where $\langle u\rangle_{\Omega}$ stands for the mean value of $u$ over $\Omega$. Hence 


$$
X^{p(\cdot)}(\Omega) \subset W^{1, p(\cdot)}(\Omega) \cap L^{2}(\Omega) .
$$

The inverse inclusion is straightforward.

Besides, by Theorem 11.2.7 of [20], it follows that

$$
W^{1, p(\cdot)}(\Omega) \cap W_{0}^{1, p^{-}}(\Omega)=W_{0}^{1, p(\cdot)}(\Omega) .
$$

Hence we conclude that

$$
\begin{aligned}
X_{0}^{p(\cdot)}(\Omega) & =X^{p(\cdot)}(\Omega) \cap W_{0}^{1, p^{-}}(\Omega) \\
& =W^{1, p(\cdot)}(\Omega) \cap L^{2}(\Omega) \cap W_{0}^{1, p^{-}}(\Omega)=W_{0}^{1, p(\cdot)}(\Omega) \cap L^{2}(\Omega) .
\end{aligned}
$$

\section{Well-posedness}

In this section, we discuss the well-posedness of (1.1)-(1.3) in an $L^{2}$-framework by using a subdifferential approach. Subdifferential is a generalized notion of functional derivative for convex functionals. Let $H$ be a Hilbert space with an inner product $(\cdot, \cdot)_{H}$ and let $\phi: H \rightarrow(-\infty, \infty]$ be a proper (i.e., $\left.\phi \not \equiv \infty\right)$ lower semicontinuous convex functional with the effective domain $D(\phi):=\{u \in$ $H ; \phi(u)<\infty\}$. Then the subdifferential operator $\partial \phi: H \rightarrow H$ of $\phi$ is defined by

$\partial \phi(u):=\left\{\xi \in H ; \phi(v)-\phi(u) \geq(\xi, v-u)_{H} \quad\right.$ for all $\left.v \in D(\phi)\right\} \quad$ for $u \in D(\phi)$ with the domain $D(\partial \phi):=\{u \in D(\phi) ; \partial \phi(u) \neq \emptyset\}$. It is well known that subdifferential operators are maximal monotone in $H$.

In [8] and [38], the well-posedness of (1.1)-(1.3) was proved by imposing the log-Hölder continuity assumption on variable exponents (see also [5]). Moreover, in [15], the well-posedness is also proved for renormalized solutions by assuming only the continuity of exponents with $1<p^{-} \leq p^{+}<N$. Here we emphasize that our well-posedness result is completely free from the continuity assumption of variable exponents, and moreover, it can cover even discontinuous variable exponents. Such a fairly general framework will be essentially required in Sect. 6 (see Remark 6.2).

Throughout this paper, we always assume that $\Omega$ is bounded and smooth. Let us begin with the definition of solutions for (1.1)-(1.3).

Definition 3.1. A function $u \in C\left([0, \infty) ; L^{2}(\Omega)\right)$ is said to be a solution of (1.1)-(1.3), if the following conditions are all satisfied:

- $u \in W_{l o c}^{1,2}\left(0, \infty ; L^{2}(\Omega)\right) \cap C\left((0, \infty) ; X_{0}^{p(\cdot)}(\Omega)\right)$ and $\Delta_{p(\cdot)} u \in L_{l o c}^{2}(0, \infty$; $\left.L^{2}(\Omega)\right)$,

- $u(0)=u_{0}$,

- For all $w \in X_{0}^{p(\cdot)}(\Omega)$, it holds that

$\int_{\Omega} \partial_{t} u(x, t) w(x) d x+\int_{\Omega}|\nabla u(x, t)|^{p(x)-2} \nabla u(x, t) \cdot \nabla w(x) d x=\int_{\Omega} f(x, t) w(x) d x$ for a.e. $t>0$. 
We reduce the initial-boundary value problem (1.1)-(1.3) into the Cauchy problem for an abstract evolution equation. Let $H:=L^{2}(\Omega)$ and define $\varphi: H \rightarrow[0, \infty]$ by

$$
\varphi(w)= \begin{cases}\int_{\Omega} \frac{1}{p(x)}|\nabla w(x)|^{p(x)} d x & \text { if } w \in X_{0}^{p(\cdot)}(\Omega), \\ \infty & \text { otherwise. }\end{cases}
$$

In order to prove the well-posedness for (1.1)-(1.3), the most crucial point lies in checking the lower semicontinuity of the functional $\varphi$ in $H=L^{2}(\Omega)$.

Lemma 3.2. Suppose that $1<p^{-}$and $p^{+}<\infty$. The function $\varphi$ is proper, convex and lower semicontinuous in $H$.

Proof. It is obvious that $\varphi$ is proper and convex in $H$. So, it remains to prove the lower semicontinuity of $\varphi$ in $H$. Let $\mu \in \mathbb{R}$ be fixed and set

$$
[\varphi \leq \mu]:=\{u \in H ; \varphi(u) \leq \mu\} .
$$

Let $\left(u_{n}\right)$ be a sequence on $[\varphi \leq \mu]$ such that $u_{n} \rightarrow u$ strongly in $H$. By Proposition 2.1, it follows that

$$
\frac{1}{p^{+}} \sigma^{-}\left(\left\|\nabla u_{n}\right\|_{p(\cdot)}\right) \leq \frac{1}{p^{+}} \int_{\Omega}\left|\nabla u_{n}(x)\right|^{p(x)} d x \leq \varphi\left(u_{n}\right) \leq \mu .
$$

Hence $\left(u_{n}\right)$ is bounded in $X_{0}^{p(\cdot)}(\Omega)$ for all $n \in \mathbb{N}$. Since $X_{0}^{p(\cdot)}(\Omega)$ is reflexive by $1<p^{-}$and $p^{+}<\infty$, we can take a subsequence of $(n)$ denoted by the same letter again such that $u_{n} \rightarrow u$ weakly in $X_{0}^{p(\cdot)}(\Omega)$.

Let $\hat{\varphi}$ be the restriction of $\varphi$ to $X_{0}^{p(\cdot)}(\Omega)$. Then $\hat{\varphi}$ is continuous in $X_{0}^{p(\cdot)}(\Omega)$, and moreover, $\hat{\varphi}$ is convex. Hence $\hat{\varphi}$ becomes weakly lower semicontinuous in $X_{0}^{p(\cdot)}(\Omega)$. Therefore we have $\liminf _{n \rightarrow \infty} \hat{\varphi}\left(u_{n}\right) \geq \hat{\varphi}(u)=\varphi(u)$, which together with the fact that $\hat{\varphi}\left(u_{n}\right)=\varphi\left(u_{n}\right) \leq \mu$ implies that $u \in[\varphi \leq \mu]$. Thus we conclude that $[\varphi \leq \mu]$ is closed in $H$, and therefore, $\varphi$ is lower semicontinuous in $H$.

One can verify the following proposition as in the constant variable case.

Proposition 3.3. The restriction $\hat{\varphi}$ of $\varphi$ to $X_{0}^{p(\cdot)}(\Omega)$ is Gâteaux differentiable, and the Gâteaux derivative $d \hat{\varphi}(u)$ of $\hat{\varphi}$ at $u$ coincides with $-\Delta_{p(\cdot)} u$ furnished with $\left.u\right|_{\partial \Omega}=0$ in the sense of distribution.

Since $\partial \varphi(u) \subset \partial \hat{\varphi}(u)=d \hat{\varphi}(u)$, we have $\partial \varphi(u)=-\Delta_{p(\cdot)} u$ with $\left.u\right|_{\partial \Omega}=0$ for all $u \in D(\partial \varphi)$. Thus the initial-boundary value problem (1.1)-(1.3) is reduced into the following Cauchy problem:

$$
\begin{gathered}
\frac{d u}{d t}(t)+\partial \varphi(u(t))=f(t) \text { in } H \text { for } t>0, \\
u(0)=u_{0} .
\end{gathered}
$$

Such an abstract evolution equation was well studied in 1970s and fundamental results were established by H. Brézis (see Chap. III of [16]). Hence we have: 
Theorem 3.4. (Well-posedness) Let $p(\cdot)$ be a measurable function from $\Omega$ into $(1, \infty)$. Assume that $1<p^{-}$and $p^{+}<\infty$. Then for all $f \in L_{l o c}^{2}\left([0, \infty) ; L^{2}(\Omega)\right)$ and $u_{0} \in L^{2}(\Omega)$, there exists a unique solution $u=u(x, t)$ of the initial-boundary value problem (1.1)-(1.3).

In particular, if $u_{0}$ belongs to $X_{0}^{p(\cdot)}(\Omega)$, then $u \in W_{l o c}^{1,2}\left([0, \infty) ; L^{2}(\Omega)\right) \cap$ $C\left([0, \infty) ; X_{0}^{p(\cdot)}(\Omega)\right)$.

Furthermore, the unique solution $u$ of (1.1)-(1.3) continuously depends on initial data $u_{0}$ and $f$ in the following sense: Let $u_{i}$ be the unique solution of (1.1)-(1.3) with $u_{0}=u_{0, i} \in L^{2}(\Omega)$ and $f=f_{i} \in L_{l o c}^{2}\left([0, \infty) ; L^{2}(\Omega)\right)$ for $i=1,2$. Then it follows that

$$
\left\|u_{1}(t)-u_{2}(t)\right\|_{2} \leq\left\|u_{0,1}-u_{0,2}\right\|_{2}+\int_{0}^{t}\left\|f_{1}(\tau)-f_{2}(\tau)\right\|_{2} d \tau \quad \text { for all } t \geq 0 .
$$

Proof. By the well-posedness result due to Brézis, the Cauchy problem (3.2), (3.3) admits a unique strong solution $u \in W_{l o c}^{1,2}\left(0, \infty ; L^{2}(\Omega)\right)$ such that $\partial \varphi(u(\cdot))$ belongs to $L_{l o c}^{2}\left(0, \infty ; L^{2}(\Omega)\right)$ and $\varphi(u(\cdot))$ is absolutely continuous in $(0, \infty)$. Here we only prove $u \in C\left((0, \infty) ; X_{0}^{p(\cdot)}(\Omega)\right)$, which is a direct consequence from a standard property of uniformly convex Banach spaces in the constant variable case.

Define a functional $\rho:\left(L^{p(\cdot)}(\Omega)\right)^{N} \rightarrow[0, \infty)$ by

$$
\rho(\mathbf{u}):= \begin{cases}\int_{\Omega} \frac{1}{p(x)}|\mathbf{u}(x)|^{p(x)} d x & \text { if } \mathbf{u} \in\left(L^{p(\cdot)}(\Omega)\right)^{N} \\ \infty & \text { else. }\end{cases}
$$

Then $\rho$ becomes a uniformly convex continuous modular in $\left(L^{p(\cdot)}(\Omega)\right)^{N}$. Since $\varphi(u(\cdot))$ is continuous in $(0, \infty)$, for each $t>0$, it follows that

$$
\rho(\nabla u(s)) \rightarrow \rho(\nabla u(t)) \quad \text { as } s \rightarrow t,
$$

and moreover, we have

$$
\nabla u(s) \rightarrow \nabla u(t) \quad \text { weakly in }\left(L^{p(\cdot)}(\Omega)\right)^{N} .
$$

Hence by Lemma 2.4.17 of [20], we deduce that

$$
\rho(\nabla u(s)-\nabla u(t)) \rightarrow 0 \quad \text { as } s \rightarrow t,
$$

which implies $\nabla u(s) \rightarrow \nabla u(t)$ strongly in $\left(L^{p(\cdot)}(\Omega)\right)^{N}$ as $s \rightarrow t$ at each $t>0$. Therefore $u \in C\left((0, \infty) ; X_{0}^{p(\cdot)}(\Omega)\right)$.

In much the same way as in the case of constant exponent $p$-Laplacians, one can also prove the comparison principle for (1.1)-(1.3). For the reader's convenience, let us show its proof.

Proposition 3.5. (Comparison principle) Assume that $p(\cdot)$ satisfies (2.1) and $p^{-}>1$. Let $u_{1}$ be a subsolution for (1.1) in the $L^{2}(\Omega)$-sense, that is, $u_{1} \in$ $C\left([0, \infty) ; L^{2}(\Omega)\right) \cap W_{l o c}^{1,2}\left((0, \infty) ; L^{2}(\Omega)\right), u_{1}(t) \in W_{0}^{1, p(\cdot)}(\Omega)$ for a.e. $t>0$, and it holds that 


$$
\int_{\Omega} \partial_{t} u_{1}(x, t) \phi(x) d x+\int_{\Omega}\left|\nabla u_{1}\right|^{p(x)-2} \nabla u_{1}(x, t) \cdot \nabla \phi(x) d x \leq \int_{\Omega} f(x, t) \phi(x) d x
$$

for every non-negative function $\phi \in W_{0}^{1, p(\cdot)}(\Omega) \cap L^{2}(\Omega)$ and for a.e. $t>0$. Let $u_{2}$ be a supersolution for (1.1) in the $L^{2}(\Omega)$-sense, which is analogously defined to the above. If $u_{1}(x, 0) \leq u_{2}(x, 0)$ for a.e. $x \in \Omega$ and $\gamma\left(u_{1}(\cdot, t)-u_{2}(\cdot, t)\right)_{+}(x)=$ 0 for a.e. $x \in \partial \Omega$ and $t>0$, where $\gamma$ stands for the trace operator from $W^{1, p^{-}}(\Omega)$ into $W^{1-1 / p^{-}, p^{-}}(\partial \Omega)$, then it follows that

$$
u_{1}(x, t) \leq u_{2}(x, t) \quad \text { for a.e. } x \in \Omega, \quad t>0 .
$$

Proof. Let $j$ be a function in $\mathbb{R}$ given by

$$
j(s)=\frac{s^{2}}{2} \text { for } s \geq 0 ; \quad j(s)=0 \text { for } s<0
$$

and define a functional $\psi$ on $H:=L^{2}(\Omega)$ by

$$
\psi(v):=\int_{\Omega} j(v(x)) d x \quad \text { for } \quad v \in H .
$$

Then $\psi$ is proper, lower semicontinuous and convex in $H$. Moreover, $\partial \psi(v)=$ $(v(\cdot))_{+} \geq 0$. Here we also note that $\partial \psi(v) \in W^{1, p(\cdot)}(\Omega)$ if $v \in W^{1, p(\cdot)}(\Omega)$.

Put $w:=u_{1}-u_{2}$. Subtracting the inequality for the supersolution $u_{2}$ from that for the subsolution $u_{1}$, we obtain

$$
\left(\frac{d w}{d t}(t), \phi\right)_{L^{2}}+\int_{\Omega}\left(\left|\nabla u_{1}\right|^{p(x)-2} \nabla u_{1}(x, t)-\left|\nabla u_{2}\right|^{p(x)-2} \nabla u_{2}(x, t)\right) \cdot \nabla \phi(x) d x
$$

$\leq 0$ for every non-negative $\phi \in W_{0}^{1, p(\cdot)}(\Omega) \cap L^{2}(\Omega)$ and for a.e. $t \geq 0$.

One can put $\phi=\partial \psi(w(t))=(w(\cdot, t))_{+}$. Indeed, since $\gamma(w(\cdot, t))_{+}(x)=0$ for a.e. $x \in \partial \Omega$ and $t>0$, we find by $(2.1)$ that $\phi \in W_{0}^{1, p(\cdot)}(\Omega)$. Applying the chain rule for subdifferentials, we get

$$
\begin{aligned}
\frac{d}{d t} & \psi(w(t)) \\
\leq & -\int_{\Omega}\left(\left|\nabla u_{1}\right|^{p(x)-2} \nabla u_{1}(x, t)-\left|\nabla u_{2}\right|^{p(x)-2} \nabla u_{2}(x, t)\right) \cdot \nabla(w(x, t))_{+} d x \\
= & -\int_{\Omega}\left(\left|\nabla u_{1}\right|^{p(x)-2} \nabla u_{1}(x, t)-\left|\nabla u_{2}\right|^{p(x)-2} \nabla u_{2}(x, t)\right) \cdot \nabla w(x, t) \\
& \quad \times \operatorname{sgn}(w(x, t)) d x \\
\leq & 0 \quad \text { for a.e. } t>0,
\end{aligned}
$$

where $\operatorname{sgn}(s)=0$ for $s \leq 0$ and $\operatorname{sgn}(s)=1$ for $s>0$. Therefore

$$
\psi(w(t)) \leq \psi(w(0))=0 \quad \text { for all } t \geq 0,
$$

which implies $w(x, t) \leq 0$ for a.e. $x \in \Omega$ and $t>0$.

Remark 3.6. In [5], the existence of periodic solutions is also proved, provided that $p^{-} \geq 2 N /(N+2)$ and $p(\cdot)$ satisfies $(2.1)$. Furthermore, it is also proved that the solution $u(t)$ converges to a stationary solution $\phi$ strongly in $L^{2}(\Omega)$ and $\varphi(u(t)) \rightarrow \varphi(\phi)$ (hence $u(t) \rightarrow \phi$ strongly in $W_{0}^{1, p(\cdot)}(\Omega)$ as $t \rightarrow \infty$ ) when 
$p^{-}>2 N /(N+2),(2.1)$ holds, $f(t) \rightarrow f_{\infty}$ weakly in $L^{2}(\Omega)$ as $t \rightarrow \infty$ and $f(\cdot)-f_{\infty} \in L^{2}\left(0, \infty ; L^{2}(\Omega)\right)$. Moreover, the convergence rate of $u(t)$ in $L^{2}(\Omega)$ is also estimated from above if $p^{-} \geq 2$.

\section{Decay and extinction properties}

In this section, we are concerned with decay and extinction properties of solutions for (1.1)-(1.3) with $f \equiv 0$ as $t \rightarrow \infty$. In case $p(\cdot)$ is constant, these properties are well known, and moreover, the optimal decay rate and the extinction rate of solutions have been revealed for the degenerate case $(p>2)$ and the fast diffusion case $(p<2)$, respectively (see $[14,18,33])$. As for the variable exponent case, Antontsev and Shmarev [7,9] also observed the extinction property of solutions without estimate for the rate of extinction when exponents are variable both in space and time. Throughout this section, the Sobolev-Poincaré inequality (2.2) plays an important role, so we always assume that

$$
p \text { satisfies }(2.1) \text { and } 2 N /(N+2) \leq p^{-}
$$

(then $X_{0}^{p(\cdot)}(\Omega)$ coincides with $W_{0}^{1, p(\cdot)}(\Omega)$, by Propositions 2.3 and 2.4).

Our main results of this section are stated in the following: For the fully degenerate case, $p^{-}>2$, it holds that

Theorem 4.1. (Decay property) In addition to (4.1), assume $p^{-}>2$. Let $u_{0} \in W_{0}^{1, p(\cdot)}(\Omega) \backslash\{0\}$ and let $u=u(x, t)$ be the unique solution of $(1.1)-(1.3)$ with $f \equiv 0$. Then there exists a constant $c>0$ such that

$$
c^{-1}(t+1)^{-1 /\left(p^{-}-2\right)} \leq\|u(t)\|_{2} \leq c(t+1)^{-1 /\left(p^{+}-2\right)} \quad \text { for all } t \geq 0 .
$$

For the fully singular case, $p^{+}<2$, we have:

Theorem 4.2. (Extinction property) In addition to (4.1), assume $p^{+}<2$. Let $u_{0} \in W_{0}^{1, p(\cdot)}(\Omega) \backslash\{0\}$ and let $u=u(x, t)$ be the unique solution of $(1.1)-(1.3)$ with $f \equiv 0$. Then there exist a finite time $t_{*}>0$ and a constant $c>0$ such that

$$
c^{-1}\left(t_{*}-t\right)_{+}^{1 /\left(2-p^{-}\right)} \leq\|u(t)\|_{2} \leq c\left(t_{*}-t\right)_{+}^{1 /\left(2-p^{+}\right)} \quad \text { for all } t \geq 0 .
$$

Hence the solution $u=u(x, t)$ vanishes at $t_{*}$, which is called extinction time of $u$.

Our proof is based on the following two fundamental energy identities:

$$
\begin{aligned}
\frac{1}{2} \frac{d}{d t}\|u(t)\|_{2}^{2}+\int_{\Omega}|\nabla u(x, t)|^{p(x)} d x & =0 \\
\left\|\frac{d u}{d t}(t)\right\|_{2}^{2}+\frac{d}{d t} \varphi(u(t)) & =0
\end{aligned}
$$

for a.e. $t \in(0, \infty)$. The first identity follows from the multiplication of (1.1) by $u$ and the integration over $\Omega$, and the second one can be obtained by multiplying (3.2) by $d u(t) / d t$ in $L^{2}(\Omega)$ and using a chain rule for subdifferentials (see Lemma 3.3 of [16]). Then we have, 
Lemma 4.3. (Upper estimates) Assume (4.1). In case $p^{+}>2$, there exists $c_{0}>0$ such that

$$
\|u(t)\|_{2} \leq c_{0}(t+1)^{-1 /\left(p^{+}-2\right)} \quad \text { for all } t \geq 0 .
$$

In case $p^{+}<2$, the solution vanishes at a finite time $t_{*}>0$.

Proof. By Proposition 2.1, it follows from (4.4) that

$$
\frac{1}{2} \frac{d}{d t}\|u(t)\|_{2}^{2}+\sigma^{-}\left(\|u(t)\|_{1, p(\cdot)}\right) \leq 0 \quad \text { for a.e. } t>0
$$

and hence, by (2.2),

$$
\frac{1}{2} \frac{d}{d t}\|u(t)\|_{2}^{2}+\sigma^{-}\left(C_{p(\cdot), 2}^{-1}\|u(t)\|_{2}\right) \leq 0 \quad \text { for a.e. } t>0 .
$$

Now, let us solve the Cauchy problem for the following ODE:

$$
y^{\prime}(t)+2 \sigma^{-}\left(C_{p(\cdot), 2}^{-1} y(t)^{1 / 2}\right)=0 \text { for } t>0, \quad y(0)=\left\|u_{0}\right\|_{2}^{2}>0 .
$$

Then by comparison principle, $\|u(t)\|_{2}^{2} \leq y(t)$ for all $t \geq 0$. In case $y(0)=$ $\left\|u_{0}\right\|_{2}^{2}>C_{p(\cdot), 2}^{2}$, one can write

$$
y^{\prime}(t)+\frac{2}{C_{p(\cdot), 2}^{p^{-}}} y(t)^{p^{-} / 2}=0 \text { for } t \in\left[0, t_{1}\right)
$$

with $t_{1}:=\sup \left\{\tau>0 ; y(t)>C_{p(\cdot), 2}^{2}\right.$ for all $\left.t \in[0, \tau]\right\}>0$. Then it follows that $\sigma^{-}\left(C_{p(\cdot), 2}^{-1} y(t)^{1 / 2}\right)=y(t)^{p^{-} / 2} / C_{p(\cdot), 2}^{p^{-}}$for $t \in\left[0, t_{1}\right)$, and hence we have,

$$
y(t)=\left\{\begin{array}{ll}
\left(\left\|u_{0}\right\|_{2}^{2-p^{-}}-\frac{2-p^{-}}{\left.C_{p(\cdot), 2}^{p^{-}} t\right)^{2 /\left(2-p^{-}\right)}}\right. & \text {if } p^{-} \neq 2, \\
\left\|u_{0}\right\|_{2}^{2} \exp \left(-\frac{2}{C_{p(\cdot), 2}^{2} t} t\right) & \text { if } p^{-}=2
\end{array} \text { for all } t \in\left[0, t_{1}\right),\right.
$$

which implies

$$
0<t_{1}<\infty \quad \text { and } \quad y\left(t_{1}\right)=C_{p(\cdot), 2}^{2}
$$

Therefore we deduce that

$$
\left\|u\left(t_{1}\right)\right\|_{2}^{2} \leq C_{p(\cdot), 2}^{2} .
$$

In case $y(0)=\left\|u_{0}\right\|_{2}^{2} \leq C_{p(\cdot), 2}^{2}$, since $y(t)$ is non-increasing by (4.7), we have

$$
y^{\prime}(t)+\frac{2}{C_{p(\cdot), 2}^{p^{+}}} y(t)^{p^{+} / 2}=0 \text { for } t \geq 0 .
$$

Then as in the last case, for any $p^{+} \neq 2$, we can obtain

$$
\|u(t)\|_{2}^{2} \leq y(t)=\left(\left\|u_{0}\right\|_{2}^{2-p^{+}}-\frac{2-p^{+}}{C_{p(\cdot), 2}^{p^{+}}} t\right)_{+}^{2 /\left(2-p^{+}\right)} \quad \text { for all } t \geq 0 .
$$


Combining these facts, we conclude that: in case $p^{+}<2$, the solution $u(t)$ vanishes at some finite time $t_{*}>0$; in case $p^{+}>2$, the solution $u(t)$ converges to zero as $t \rightarrow \infty$ such that

$$
\|u(t)\|_{2} \leq c_{0}(t+1)^{-1 /\left(p^{+}-2\right)} \quad \text { for all } t \geq 0
$$

with some constant $c_{0}>0$. This completes our proof.

In constant exponent cases, one can use the fact that the Rayleigh quotient $t \mapsto R(t):=\|\nabla u(t)\|_{p} /\|u(t)\|_{2}$ is non-increasing. However, in variable exponent cases, its analogue is not obvious, because of a gap between the norm $\|\nabla w\|_{p(\cdot)}$ and the modular $\rho(w)=\int_{\Omega}|\nabla w(x)|^{p(x)} d x$ in $L^{p(\cdot)}(\Omega)$. Here, to derive the lower and upper estimates for $\|u(t)\|_{2}$, we set up the following lemma, where a modified Rayleigh quotient involving a Lyapunov energy $\varphi$ is introduced.

Lemma 4.4. The function

$$
t \mapsto \tilde{R}(t):=\frac{\varphi(u(t))}{\|u(t)\|_{2}^{p^{-}}}
$$

is non-increasing on the interval $I:=\left\{t \geq 0 ;\|u(t)\|_{2}>0\right\}$.

Proof. By (4.5), it follows that

$$
\frac{d}{d t} \tilde{R}(t)=\frac{-\left\|u^{\prime}(t)\right\|_{2}^{2}\|u(t)\|_{2}^{p^{-}}-\varphi(u(t)) \frac{d}{d t}\|u(t)\|_{2}^{p^{-}}}{\|u(t)\|_{2}^{2 p^{-}}} \quad \text { for all } t \in I .
$$

Here we note by (4.4) that

$$
\frac{d}{d t}\|u(t)\|_{2}^{p^{-}}=\frac{p^{-}}{2}\|u(t)\|_{2}^{p^{-}-2} \frac{d}{d t}\|u(t)\|_{2}^{2} .
$$

Hence

$$
\frac{d}{d t} \tilde{R}(t)=\frac{-\left\|u^{\prime}(t)\right\|_{2}^{2}\|u(t)\|_{2}^{2}-\varphi(u(t)) \frac{p^{-}}{2} \frac{d}{d t}\|u(t)\|_{2}^{2}}{\|u(t)\|_{2}^{p^{-}+2}}
$$

By (4.4),

$$
\begin{aligned}
\varphi(u(t)) & =\int_{\Omega} \frac{1}{p(x)}|\nabla u(x, t)|^{p(x)} d x \\
& \leq \frac{1}{p^{-}} \int_{\Omega}|\nabla u(x, t)|^{p(x)} d x=-\frac{1}{2 p^{-}} \frac{d}{d t}\|u(t)\|_{2}^{2} .
\end{aligned}
$$

Thus since $(d / d t)\|u(t)\|_{2}^{2} \leq 0$ by (4.4), it holds that

$$
\begin{aligned}
\frac{d}{d t} \tilde{R}(t) & \leq \frac{-\left\|u^{\prime}(t)\right\|_{2}^{2}\|u(t)\|_{2}^{2}+\left(\frac{1}{2} \frac{d}{d t}\|u(t)\|_{2}^{2}\right)^{2}}{\|u(t)\|_{2}^{p^{-}+2}} \\
& =\frac{-\left\|u^{\prime}(t)\right\|_{2}^{2}\|u(t)\|_{2}^{2}+\left(u^{\prime}(t), u(t)\right)_{L^{2}}^{2}}{\|u(t)\|_{2}^{p^{-}+2}} \quad \text { for all } t \in I,
\end{aligned}
$$

which implies that 


$$
\frac{d}{d t} \tilde{R}(t) \leq 0 \quad \text { for all } t \in I
$$

Therefore the function $\tilde{R}(\cdot)$ is non-increasing on $I$.

Now, we are in a position to give a proof of Theorem 4.1.

Proof of Theorem 4.1. The upper estimate has already been proved in Lemma 4.3. Hence let us prove the lower estimate. Recalling (4.4) and using the definition of $p^{+}$, we find that

$$
\frac{d}{d t}\|u(t)\|_{2}^{2}+2 p^{+} \varphi(u(t)) \geq 0 \text { for all } t \geq 0 .
$$

By Lemma 4.4, we note that

$$
\varphi(u(t))=\tilde{R}(t)\|u(t)\|_{2}^{p^{-}} \leq \tilde{R}(0)\|u(t)\|_{2}^{p^{-}} \quad \text { if }\|u(t)\|_{2}>0 .
$$

Hence we obtain

$$
\frac{d}{d t}\|u(t)\|_{2}^{2}+2 p^{+} \tilde{R}(0)\|u(t)\|_{2}^{p^{-}} \geq 0 \quad \text { for a.e. } t>0 .
$$

By solving the following ODE:

$$
y^{\prime}(t)+\kappa^{-} y(t)^{p^{-} / 2}=0 \text { for } t>0, \quad y(0)=\left\|u_{0}\right\|_{2}^{2}>0
$$

with $\kappa^{-}:=2 p^{+} \tilde{R}(0)$ and by using the comparison principle, we deduce by $p^{-}>2$ that

$$
\|u(t)\|_{2} \geq\left(\left\|u_{0}\right\|_{2}^{2-p^{-}}+\left(p^{-}-2\right) p^{+} \tilde{R}(0) t\right)^{-1 /\left(p^{-}-2\right)} \quad \text { for all } t \geq 0,
$$

which implies our desired result.

We next prove Theorem 4.2.

Proof of Theorem 4.2. Let us derive the upper estimate for $\|u(t)\|_{2}$. As in the proof of Theorem 4.1, by the definition of $p^{+}$and Lemma 4.4, one can obtain

$$
\frac{d}{d t}\|u(t)\|_{2}^{2}+2 p^{+} \tilde{R}(0)\|u(t)\|_{2}^{p^{-}} \geq 0 \quad \text { for a.e. } t \in\left(0, t_{*}\right)
$$

which implies

$$
\frac{2}{2-p^{-}} \frac{d}{d t}\|u(t)\|_{2}^{2-p^{-}} \geq-2 p^{+} \tilde{R}(0) .
$$

The integration of both sides over $\left(t, t_{*}\right)$ leads us to

$$
\|u(t)\|_{2} \leq\left\{\left(2-p^{-}\right) p^{+} \tilde{R}(0)\right\}^{1 /\left(2-p^{-}\right)}\left(t_{*}-t\right)_{+}^{1 /\left(2-p^{-}\right)} \quad \text { for all } t \geq 0 .
$$

We next prove the lower estimate. Set $t_{1}:=\inf \left\{\tau \geq 0 ;\|u(\tau)\|_{2} \leq C_{p(\cdot), 2}\right\}$. Then $0 \leq t_{1}<t_{*}$ and $\sigma^{-}\left(C_{p(\cdot), 2}^{-1}\|u(t)\|_{2}\right)=\|u(t)\|_{2}^{p^{+}} / C_{p(\cdot), 2}^{p^{+}}$for all $t \geq t_{1}$. Moreover, by (4.8) in the proof of Lemma 4.3, we find that

$$
0 \leq t_{1} \leq\left(\frac{C_{p(\cdot), 2}^{p^{+}}\left\|u_{0}\right\|_{2}^{2-p^{+}}-C_{p(\cdot), 2}^{2}}{2-p^{+}}\right)_{+}
$$


It then follows from (4.6) that

$$
\frac{2}{2-p^{+}} \frac{d}{d t}\|u(t)\|_{2}^{2-p^{+}} \leq-\frac{2}{C_{p(\cdot), 2}^{p^{+}}} \quad \text { for a.e. } t>t_{1} .
$$

Integrating both sides over $\left(t, t_{*}\right)$, we have

$$
\|u(t)\|_{2} \geq\left(\frac{2-p^{+}}{C_{p(\cdot), 2}^{p^{+}}}\right)^{1 /\left(2-p^{+}\right)}\left(t_{*}-t\right)_{+}^{1 /\left(2-p^{+}\right)} \quad \text { for all } t \geq t_{1} .
$$

As for the case that $t_{1}>0$ (hence $\left.\left\|u\left(t_{1}\right)\right\|_{2}=C_{p(\cdot), 2}\right)$, taking a positive constant $c_{1}:=\left\|u\left(t_{1}\right)\right\|_{2} / t_{*}^{1 /\left(2-p^{+}\right)}$, we observe

$$
\|u(t)\|_{2} \geq\left\|u\left(t_{1}\right)\right\|_{2}=c_{1} t_{*}^{1 /\left(2-p^{+}\right)} \geq c_{1}\left(t_{*}-t\right)^{1 /\left(2-p^{+}\right)} \quad \text { for all } t \in\left[0, t_{1}\right] .
$$

Thus we have proved this theorem.

One can also obtain the following corollary, which provides some estimate for the extinction time in terms of initial data.

Corollary 4.5. (Estimates for extinction times) In addition to (4.1), assume that $p^{+}<2$. For each $u_{0} \in W_{0}^{1, p(\cdot)}(\Omega) \backslash\{0\}$ and $f \equiv 0$, let $u=u(x, t)$ be the unique solution of (1.1)-(1.3) with the extinction time $t_{*}=t_{*}\left(u_{0}\right)>0$. Then it follows that

$$
\frac{1}{2-p^{-}} \frac{\left\|u_{0}\right\|_{2}^{2}}{p^{+} \varphi\left(u_{0}\right)} \leq t_{*}\left(u_{0}\right) \leq \frac{C_{p(\cdot), 2}^{p^{+}}}{2-p^{+}}\left\|u_{0}\right\|_{2}^{2-p^{+}} .
$$

Proof. The lower estimate follows immediately from (4.10) with $t=0$. Moreover, recall that

$$
\left\|u\left(t_{1}\right)\right\|_{2} \leq C_{p(\cdot), 2},
$$

where $t_{1}$ is estimated by (4.11). Substitute $t=t_{1}$ in (4.12) to get

$$
\left\|u\left(t_{1}\right)\right\|_{2} \geq\left(\frac{2-p^{+}}{C_{p(\cdot), 2}^{p^{+}}}\right)^{1 /\left(2-p^{+}\right)}\left(t_{*}-t_{1}\right)^{1 /\left(2-p^{+}\right)},
$$

which together with (4.11) implies the upper estimate.

Remark 4.6. One can also estimate the $W_{0}^{1, p(\cdot)}(\Omega)$-norm of $u(t)$ from above and below by using (2.2) and the fact that

$$
\sigma^{-}\left(\|u(t)\|_{1, p(\cdot)}\right) \leq p^{+} \varphi(u(t)) \leq p^{+} \frac{\varphi\left(u_{0}\right)}{\left\|u_{0}\right\|_{2}^{p^{-}}}\|u(t)\|_{2}^{p^{-}} .
$$

Then in case $p^{-}>2$, it follows that

$\tilde{c}^{-1}(t+1)^{-1 /\left(p^{-}-2\right)} \leq\|u(t)\|_{1, p(\cdot)}$ and $\sigma^{-}\left(\|u(t)\|_{1, p(\cdot)}\right)^{1 / p^{-}} \leq \tilde{c}(t+1)^{-1 /\left(p^{+}-2\right)} ;$ 
in case $p^{+}<2$, we have

$\tilde{c}^{-1}\left(t_{*}-t\right)_{+}^{1 /\left(2-p^{-}\right)} \leq\|u(t)\|_{1, p(\cdot)}$ and $\sigma^{-}\left(\|u(t)\|_{1, p(\cdot)}\right)^{1 / p^{-}} \leq \tilde{c}\left(t_{*}-t\right)_{+}^{1 /\left(2-p^{+}\right)}$

with some constant $\tilde{c}>0$. Since $\sigma^{-}(s)=s^{p^{+}}$for all $s \in[0,1]$, the decay rate (resp., the extinction rate) will be equal to or faster than $(t+1)^{-p^{-} /\left\{p^{+}\left(p^{+}-2\right)\right\}}$ as $t \rightarrow \infty$ (resp., $\left(t_{*}-t\right)^{p^{-} /\left\{p^{+}\left(2-p^{+}\right)\right\}}$as $t \rightarrow t_{*}$ ).

\section{Fast/slow diffusion limit}

Let $\left(p_{n}(\cdot)\right)$ be a sequence of measurable functions from $\Omega$ into $(1, \infty)$ such that

$$
p_{n}(x) \rightarrow \infty \quad \text { for a.e. } x \in \Omega \text {. }
$$

In this section, we shall investigate the limiting behavior as $n \rightarrow \infty$ of the solutions $u_{n}=u_{n}(x, t)$ for

$$
\begin{aligned}
\partial_{t} u_{n}=\Delta_{p_{n}(\cdot)} u_{n}+f_{n} & \text { in } \Omega \times(0, T), \\
u_{n}=0 & \text { on } \partial \Omega \times(0, T), \\
u_{n}(\cdot, 0)=u_{0, n} & \text { in } \Omega
\end{aligned}
$$

with $T>0$ and sequences $\left(u_{0, n}\right)$ and $\left(f_{n}\right)$ in $L^{2}(\Omega)$ and $L^{2}\left(0, T ; L^{2}(\Omega)\right)$, respectively, satisfying

$$
\begin{aligned}
u_{0, n} \rightarrow u_{0} & \text { strongly in } L^{2}(\Omega), \\
f_{n} \rightarrow f & \text { strongly in } L^{2}\left(0, T ; L^{2}(\Omega)\right) .
\end{aligned}
$$

The limit of solutions $u_{n}$ as $n \rightarrow \infty$ has been studied in the constant exponent case, i.e., $p_{n}(\cdot) \equiv p_{n}$, and such a problem arises from a critical-state model of type-II superconductors (see [4,6,13]) and a growing sandpile model (see [11]). Particularly, in [4], the first author characterized the limit of solutions for (5.1)-(5.3) by employing the notion of Mosco convergence of a sequence $\left(\psi_{n}\right)$ of functionals associated with $p_{n}$-Laplacians:

$$
\psi_{n}(w):= \begin{cases}\frac{1}{p_{n}} \int_{\Omega}|\nabla w(x)|^{p_{n}} d x & \text { if } w \in W_{0}^{1, p_{n}}(\Omega), \\ \infty & \text { otherwise }\end{cases}
$$

and by exploiting a general theory for the convergence as $n \rightarrow \infty$ of solutions for abstract evolution equations governed by subdifferential operators $\partial \psi_{n}$ of $\psi_{n}$ in a Hilbert space $H$ :

$$
\frac{d u_{n}}{d t}(t)+\partial \psi_{n}\left(u_{n}(t)\right) \ni f_{n}(t) \text { in } H \text { for } t \in(0, T), \quad u_{n}(0)=u_{0, n} .
$$

In this section we also follow the same strategy to investigate the limit of solutions for $(5.1)-(5.3)$ with the sequence $\left(p_{n}(\cdot)\right)$ of variable exponents as $n \rightarrow \infty$. Before starting our analysis let us briefly review the notion of Mosco convergence and recall the convergence result due to Attouch [12] for evolution equations governed by subdifferential operators in the next subsection. 


\subsection{Mosco convergence and evolution equations}

The Mosco convergence of convex functionals is defined as follows:

Definition 5.1. (Mosco convergence) Let $H$ be a Hilbert space and denote by $\Phi(H)$ the set of all proper (i.e., $\phi \not \equiv \infty)$, lower semicontinuous and convex functionals $\phi$ from $H$ into $(-\infty, \infty]$. Let $\left(\phi_{n}\right)$ be a sequence in $\Phi(H)$ and let $\phi \in \Phi(H)$. Then $\phi_{n} \rightarrow \phi$ on $H$ in the sense of Mosco as $n \rightarrow \infty$ if the following conditions are all satisfied:

(i) For all $u \in D(\phi)$, there exists a sequence $\left(u_{n}\right)$ in $H$ such that $u_{n} \rightarrow u$ strongly in $H$ and $\phi_{n}\left(u_{n}\right) \rightarrow \phi(u)$.

(ii) Let $\left(u_{n}\right)$ be a sequence in $H$ such that $u_{n} \rightarrow u$ weakly in $H$. Then $\liminf _{n \rightarrow \infty} \phi_{n}\left(u_{n}\right) \geq \phi(u)$.

Attouch [12] investigated the limit as $n \rightarrow \infty$ of solutions for the following Cauchy problems:

$$
\begin{gathered}
\frac{d u_{n}}{d t}(t)+\partial \phi_{n}\left(u_{n}(t)\right) \ni f_{n}(t) \text { in } H \text { for } t \in(0, T), \\
u_{n}(0)=u_{0, n} .
\end{gathered}
$$

Proposition 5.2. (Theorem 3.74 of [12]) Let $\phi_{n}, \phi \in \Phi(H)$ be such that

$$
\phi_{n} \rightarrow \phi \text { on } H \text { in the sense of Mosco as } n \rightarrow+\infty \text {. }
$$

Moreover, let $f_{n}, f \in L^{2}(0, T ; H)$ be such that

$$
f_{n} \rightarrow f \quad \text { strongly in } L^{2}(0, T ; H)
$$

and let $u_{0, n} \in \overline{D\left(\phi_{n}\right)}$ and $u_{0} \in \overline{D(\phi)}$ be such that

$$
u_{0, n} \rightarrow u_{0} \quad \text { strongly in } H \text {. }
$$

Then the solutions $u_{n}$ of (5.4), (5.5) converge to $u$ as $n \rightarrow \infty$ in the following sense:

$$
\begin{array}{r}
u_{n} \rightarrow u \quad \text { strongly in } C([0, T] ; H), \\
\sqrt{t} \frac{d u_{n}}{d t} \rightarrow \sqrt{t} \frac{d u}{d t} \quad \text { strongly in } L^{2}(0, T ; H) .
\end{array}
$$

Moreover, the limit $u$ is the unique solution of

$$
\frac{d u}{d t}(t)+\partial \phi(u(t)) \ni f(t) \quad \text { in } H, 0<t<T, \quad u(0)=u_{0} .
$$

In addition, if $\phi_{n}\left(u_{0, n}\right) \rightarrow \phi\left(u_{0}\right)<\infty$, then

$$
\begin{aligned}
u_{n} \rightarrow u & \text { strongly in } W^{1,2}(0, T ; H), \\
\phi_{n}\left(u_{n}(\cdot)\right) \rightarrow \phi(u(\cdot)) & \text { uniformly on }[0, T] .
\end{aligned}
$$

\subsection{Limit of solutions for the $p_{n}(\cdot)$-Laplacian}

Let us return to our issue of the fast/slow diffusion limit. Here and thereafter, we write

$$
p_{n}^{+}:=\underset{x \in \Omega}{\operatorname{ess} \sup _{n}} p_{n}(x), \quad p_{n}^{-}:=\operatorname{ess} \inf _{x \in \Omega} p_{n}(x) .
$$

Our result is stated as follows: 
Theorem 5.3. (Convergence of solutions) Let $\left(p_{n}(\cdot)\right)$ be a sequence of measurable functions from $\Omega$ into $(1, \infty)$ such that

$$
p_{n}^{-} \rightarrow \infty \quad \text { and } \quad\left(p_{n}^{+}\right)^{1 / p_{n}^{-}} \rightarrow 1 \quad \text { as } n \rightarrow \infty,
$$

where the latter is equivalently rewritten into

$$
\frac{\log p_{n}^{+}}{p_{n}^{-}} \rightarrow 0 \quad \text { as } n \rightarrow \infty
$$

Let $f_{n} \in L^{2}\left(0, T ; L^{2}(\Omega)\right)$ and $u_{0, n} \in L^{2}(\Omega)$ be such that

$$
\begin{aligned}
f_{n} \rightarrow f & \text { strongly in } L^{2}\left(0, T ; L^{2}(\Omega)\right), \\
u_{0, n} \rightarrow u_{0} & \text { strongly in } L^{2}(\Omega)
\end{aligned}
$$

and let $u_{n}$ be the solutions of (5.1)-(5.3). Then there exists a function $u \in$ $C\left([0, T] ; L^{2}(\Omega)\right) \cap W_{l o c}^{1,2}\left((0, T] ; L^{2}(\Omega)\right)$ such that

$$
\begin{aligned}
& \int_{\Omega}\left(f(x, t)-\partial_{t} u(x, t)\right)(v(x)-u(x, t)) d x \leq 0 \quad \text { for a.e. } t \in(0, T), \\
& \quad \text { and all } v \in K, \\
& u(t) \in K \quad \text { for a.e. } t \in(0, T) \quad \text { and } \quad u(\cdot, 0)=u_{0} \quad \text { in } \Omega,
\end{aligned}
$$

where $K:=\left\{v \in H_{0}^{1}(\Omega) ;\|\nabla v\|_{\infty} \leq 1\right\}$, and

$$
\begin{array}{r}
u_{n} \rightarrow u \quad \text { strongly in } C\left([0, T] ; L^{2}(\Omega)\right), \\
\sqrt{t} \frac{d u_{n}}{d t} \rightarrow \sqrt{t} \frac{d u}{d t} \quad \text { strongly in } L^{2}\left(0, T ; L^{2}(\Omega)\right) .
\end{array}
$$

In addition, if $\int_{\Omega}\left(1 / p_{n}(x)\right)\left|\nabla u_{0, n}(x)\right|^{p_{n}(x)} d x \rightarrow 0$, then it also holds that

$$
u_{n} \rightarrow u \quad \text { strongly in } W^{1,2}\left(0, T ; L^{2}(\Omega)\right) \text {. }
$$

Remark 5.4. (Comparison to an elliptic case) In [31], Manfredi et al. studied the limit of solutions $u_{n}=u_{n}(x)$ for the Dirichlet problem

$$
-\Delta_{p_{n}(\cdot)} u_{n}=0 \text { in } \Omega, \quad u_{n}=f \text { on } \partial \Omega
$$

with some given function $f=f(x)$. They proved that $u_{n}$ uniformly converges to the unique viscosity solution $u$ of

$$
-\Delta_{\infty} u-|\nabla u|^{2} \ln |\nabla u| \boldsymbol{\xi} \cdot \nabla u=0 \text { in } \Omega, \quad u=f \text { on } \partial \Omega,
$$

where $\Delta_{\infty} u=\left(D^{2} u \nabla u\right) \cdot \nabla u$, under the assumption that

$$
\frac{\nabla p_{n}(x)}{p_{n}(x)} \rightarrow \boldsymbol{\xi}(x) \quad \text { uniformly in } \Omega,
$$

which always implies (5.6). In this elliptic case, in order to avoid the divergence of each term as $p_{n}(x) \rightarrow \infty$, one can carry out a normalization of the expanded form of the equation,

$$
\begin{aligned}
0= & \Delta_{p_{n}(\cdot)} u_{n}(x) \\
= & \left|\nabla u_{n}(x)\right|^{p_{n}(x)-2} \Delta u_{n}(x)+\left(p_{n}(x)-2\right)\left|\nabla u_{n}(x)\right|^{p_{n}(x)-4} \Delta_{\infty} u_{n}(x) \\
& +\left|\nabla u_{n}(x)\right|^{p_{n}(x)-2} \ln \left|\nabla u_{n}(x)\right| \nabla p_{n}(x) \cdot \nabla u_{n}(x),
\end{aligned}
$$


by dividing both sides by $p_{n}(x)\left|\nabla u_{n}(x)\right|^{p_{n}(x)-4}$ beforehand. On the other hand, in parabolic cases, such a normalization cannot be applied due to the presence of the term $\partial_{t} u$. Then the limiting problem is essentially different from that of the elliptic case, and it is described as a variational inequality.

To prove this theorem, define the functionals $\varphi_{n}: H:=L^{2}(\Omega) \rightarrow[0, \infty]$ by

$$
\varphi_{n}(w)= \begin{cases}\int_{\Omega} \frac{1}{p_{n}(x)}|\nabla w(x)|^{p_{n}(x)} d x & \text { if } w \in X_{0}^{p_{n}(\cdot)}(\Omega), \\ \infty & \text { otherwise. }\end{cases}
$$

Then as in Sect. 3, problem (5.1)-(5.3) is transcribed into the Cauchy problem (5.4), (5.5) with $\phi_{n}$ replaced by $\varphi_{n}$ in $H=L^{2}(\Omega)$.

We next verify the Mosco convergence of $\varphi_{n}$ as $p_{n}(\cdot) \rightarrow \infty$ to a convex function $\varphi_{\infty}$ on $L^{2}(\Omega)$ under an appropriate control of the fastest and slowest speeds of $p_{n}(\cdot) \rightarrow \infty$ over $\Omega$.

Proposition 5.5. (Mosco convergence) Under the same assumptions of $p_{n}(\cdot)$ as in Theorem 5.3, $\varphi_{n}$ converges to $\varphi_{\infty}$ on $L^{2}(\Omega)$ in the sense of Mosco as $n \rightarrow \infty$, where $\varphi_{\infty}$ denotes the indicator function over the closed convex subset of $L^{2}(\Omega)$,

$$
K:=\left\{u \in H_{0}^{1}(\Omega) ;\|\nabla u\|_{\infty} \leq 1\right\}
$$

that is, $\varphi_{\infty}$ is a function from $L^{2}(\Omega)$ to $[0, \infty]$ given by

$$
\varphi_{\infty}(w):= \begin{cases}0 & \text { if } w \in K \\ \infty & \text { otherwise. }\end{cases}
$$

Proof. Let $u \in D\left(\varphi_{\infty}\right)=K$ be fixed. We then set a sequence $u_{n} \equiv u$ in $D\left(\varphi_{\infty}\right)$ and observe that

$$
0 \leq \varphi_{n}\left(u_{n}\right)=\int_{\Omega} \frac{1}{p_{n}(x)}|\nabla u(x)|^{p_{n}(x)} d x \leq \frac{|\Omega|}{p_{n}^{-}} \rightarrow 0 \quad \text { as } n \rightarrow \infty .
$$

Here we also used the fact that $u_{n}=u \in D\left(\varphi_{n}\right)$ for each $n \in \mathbb{N}$. Indeed, since $u \in H_{0}^{1}(\Omega)$ and $\nabla u \in\left(L^{\infty}(\Omega)\right)^{N}$, we have $u \in W_{0}^{1, r}(\Omega)$ for any $r \in[1, \infty)$, which implies $u \in X_{0}^{p_{n}(\cdot)}(\Omega)=D\left(\varphi_{n}\right)$. Hence $\varphi_{n}\left(u_{n}\right) \rightarrow \varphi_{\infty}(u)$ as $n \rightarrow \infty$. Thus (i) of Definition 5.1 follows.

As for (ii) of Definition 5.1, let $\left(u_{n}\right)$ be a sequence in $L^{2}(\Omega)$ such that $u_{n} \rightarrow u$ weakly in $L^{2}(\Omega)$. We then claim that

$$
\liminf _{n \rightarrow \infty} \varphi_{n}\left(u_{n}\right) \geq \varphi_{\infty}(u) .
$$

Indeed, for the case where the liminf of $\varphi_{n}\left(u_{n}\right)$ is infinite, (5.13) follows immediately. For the case where the liminf is finite, up to a subsequence, it follows that

$$
\varphi_{n}\left(u_{n}\right) \leq C
$$


for some constant $C$ independent of $n$. Then we observe that

$$
\begin{aligned}
1 \geq \frac{\varphi_{n}\left(u_{n}\right)}{C} & =\int_{\Omega}\left\{\frac{1}{\left(p_{n}(x) C\right)^{1 / p_{n}(x)}}\left|\nabla u_{n}(x)\right|\right\}^{p_{n}(x)} d x \\
& \geq \int_{\Omega}\left\{\frac{1}{\left(p_{n}^{+} C\right)^{1 / p_{n}^{-}}}\left|\nabla u_{n}(x)\right|\right\}^{p_{n}(x)} d x \quad \text { for large } n,
\end{aligned}
$$

which implies

$$
\left\|\nabla u_{n}\right\|_{p_{n}(\cdot)} \leq\left(p_{n}^{+} C\right)^{1 / p_{n}^{-}} .
$$

Thus, since $p_{n}^{-}-1<p_{n}(x)$ for a.e. $x \in \Omega$, by Hölder's inequality (see Lemma 3.2 .20 of $[20])$, we have

$$
\int_{\Omega}\left|\nabla u_{n}(x)\right|^{p_{n}^{-}-1} d x \leq 2\left\|\left|\nabla u_{n}(x)\right|^{p_{n}^{-}-1}\right\|_{p_{n}(\cdot) /\left(p_{n}^{-}-1\right)}\|1\|_{r_{n}(\cdot)},
$$

where $r_{n}: \Omega \rightarrow(1, \infty)$ is defined by

$$
\frac{p_{n}^{-}-1}{p_{n}(x)}+\frac{1}{r_{n}(x)}=1
$$

Here we observe

$$
\begin{aligned}
& \left\|\left|\nabla u_{n}(x)\right|^{p_{n}^{-}-1}\right\|_{p_{n}(\cdot) /\left(p_{n}^{-}-1\right)} \\
& \quad=\inf \left\{\lambda>0 ; \int_{\Omega}\left(\frac{\left|\nabla u_{n}(x)\right|}{\lambda^{1 /\left(p_{n}^{-}-1\right)}}\right)^{p_{n}(x)} d x \leq 1\right\}=\left\|\nabla u_{n}\right\|_{p_{n}(\cdot)}^{p^{-}-1} .
\end{aligned}
$$

Moreover, since $r_{n}(\cdot)=p_{n}(\cdot) /\left(p_{n}(\cdot)-p_{n}^{-}+1\right)>1$, we find that $\|1\|_{r_{n}(\cdot)} \leq$ $\max \{1,|\Omega|\}$ (see Lemma 3.2.11 of $[20]$ ). Therefore it follows that

$$
\begin{aligned}
\int_{\Omega}\left|\nabla u_{n}(x)\right|^{p_{n}^{-}-1} d x & \leq 2 \max \{1,|\Omega|\}\left\|\nabla u_{n}\right\|_{p_{n}(\cdot)}^{p_{n}^{-}-1} \\
& \leq 2 \max \{1,|\Omega|\}\left(p_{n}^{+} C\right)^{\left(p_{n}^{-}-1\right) / p_{n}^{-}} .
\end{aligned}
$$

On the other hand, it follows that

$$
\left\|\nabla u_{n}\right\|_{q} \leq(|\Omega|+1)^{1 / q}\left\|\nabla u_{n}\right\|_{p_{\bar{n}}^{-}-1}
$$

for an arbitrary $q \in\left[1, p_{n}^{-}-1\right)$. Since the right-hand side is bounded in $n$ by (5.14) and assumption, for each $q>1$, up to a subsequence, we see

$$
\nabla u_{n} \rightarrow \nabla u \quad \text { weakly in }\left(L^{q}(\Omega)\right)^{N} \quad \text { as } n \rightarrow \infty
$$

and $u \in H_{0}^{1}(\Omega)$. Hence passing to the limit as $p_{n}^{-} \rightarrow \infty$ with the assumption that $\left(p_{n}^{+}\right)^{1 / p_{n}^{-}} \rightarrow 1$, we derive

$$
\begin{aligned}
\|\nabla u\|_{q} & \leq \liminf _{n \rightarrow \infty}\left\|\nabla u_{n}\right\|_{q} \\
& \leq \lim _{n \rightarrow \infty}(|\Omega|+1)^{1 / q}(2 \max \{1,|\Omega|\})^{1 /\left(p_{n}^{-}-1\right)}\left(p_{n}^{+} C\right)^{1 / p_{n}^{-}} \\
& =(|\Omega|+1)^{1 / q} .
\end{aligned}
$$


Then letting $q \rightarrow \infty$, we find that

$$
\|\nabla u\|_{\infty} \leq 1
$$

Thus it follows by $\varphi_{n} \geq 0$ that

$$
\varphi_{\infty}(u)=0 \leq \liminf _{n \rightarrow \infty} \varphi_{n}\left(u_{n}\right)
$$

Therefore (5.13) holds. Consequently, $\varphi_{n} \rightarrow \varphi_{\infty}$ on $L^{2}(\Omega)$ in the sense of Mosco as $n \rightarrow \infty$.

We are now in position to prove Theorem 5.3.

Proof of Theorem 5.3. Due to Propositions 5.2 and 5.5, the solutions $u_{n}$ converge to a limit $u$ and it uniquely solves

$$
\frac{d u}{d t}(t)+\partial \varphi_{\infty}(u(t)) \ni f(t), \quad 0<t<T, \quad u(0)=u_{0} .
$$

From the definition of subdifferential, the evolution equation above can be rewritten by the following variational inequality:

$\left(f(t)-\frac{d u}{d t}(t), v-u(t)\right)_{L^{2}} \leq \varphi_{\infty}(v)-\varphi_{\infty}(u(t))=0$ for all $v \in D\left(\varphi_{\infty}\right)=K$ and $u(t) \in K$ for a.e. $t \in(0, T)$.

\section{Partial fast/slow diffusion limit}

We finally discuss the case that $p_{n}(\cdot)$ diverges only in a subset of $\Omega$ as $n \rightarrow \infty$. More precisely, we address ourselves to the following case:

$$
p_{n}(x)= \begin{cases}q_{n}(x) \rightarrow \infty & \text { if } x \in D, \\ q(x)<\infty & \text { if } x \in \Omega \backslash \bar{D} \quad \text { as } n \rightarrow \infty\end{cases}
$$

where $D$ is a non-empty open subset of $\Omega$ satisfying $|D|,|\Omega \backslash \bar{D}|>0$ and $q: \Omega \backslash \bar{D} \rightarrow(1, \infty)$ and $q_{n}: D \rightarrow(1, \infty)$ are measurable, and we shall discuss the convergence of solutions for (5.1)-(5.3) as $n \rightarrow \infty$. In this case, the limiting problem will be described as a mixture of two problems, a nonlinear diffusion equation involving the $q(\cdot)$-Laplacian in $\Omega \backslash \bar{D}$ and an evolutionary quasi-variational inequality over $D$. Moreover, it is noteworthy that the constraint set of the quasi-variational inequality depends on the unknown function (cf. the set $K$ is independent of $u$ in Sect. 5).

Our basic strategy here is also based on the Mosco convergence of the functionals $\varphi_{n}$ associated with $p_{n}(\cdot)$-Laplacians as in the last section (see (5.12)). Throughout this section, we write

$$
\begin{array}{cl}
q^{+} & :=\operatorname{essup}_{x \in \Omega \backslash \bar{D}} q(x) \quad \text { and } \quad q^{-}:=\underset{x \in \Omega \backslash \bar{D}}{\operatorname{essinf}} q(x), \\
q_{n}^{+}:=\operatorname{ess}_{x \in D} q_{n}(x) \quad \text { and } \quad q_{n}^{-}:=\operatorname{essinf}_{x \in D} q_{n}(x)
\end{array}
$$

and suppose that

$$
1<q_{n}^{-}, q^{-} \text {and } q_{n}^{+}, q^{+}<\infty \quad \text { for all } n \in \mathbb{N} .
$$


For each function $w: \Omega \rightarrow \mathbb{R}$, we simply use the same letter $w$ for the restriction of $w$ onto a subset of $\Omega$ if no confusion can arise.

Now, our main result here reads,

Theorem 6.1. (Convergence of solutions) Let $\left(p_{n}(\cdot)\right)$ be a sequence given in (6.1) such that (6.2) and the following hold:

$$
q_{n}^{-} \rightarrow \infty \quad \text { and } \quad\left(q_{n}^{+}\right)^{1 / q_{n}^{-}} \rightarrow 1 \quad \text { as } n \rightarrow \infty .
$$

Moreover, let $f_{n} \in L^{2}\left(0, T ; L^{2}(\Omega)\right)$ and $u_{0, n} \in L^{2}(\Omega)$ be such that (5.7) and (5.8) hold. Let $u_{n}$ be the solutions of (5.1)-(5.3). Then there exists a function $u \in C\left([0, T] ; L^{2}(\Omega)\right) \cap W_{l o c}^{1,2}\left((0, T] ; L^{2}(\Omega)\right)$ such that (5.9) and (5.10) hold, and moreover, the limit $u$ satisfies

$$
\begin{gathered}
u(t) \in W_{0}^{1, q^{-}}(\Omega), \quad u(t) \in X^{q(\cdot)}(\Omega \backslash \bar{D}) \quad \text { for a.e. } t \in(0, T), \\
u(\cdot, 0)=u_{0} \quad \text { in } \Omega
\end{gathered}
$$

and solves the following mixed problem for a.e. $t \in(0, T)$ : a nonlinear diffusion equation on $\Omega \backslash \bar{D}$ driven by the $q(\cdot)$-Laplacian,

$$
\partial_{t} u(\cdot, t)-\Delta_{q(\cdot)} u(\cdot, t)=f(\cdot, t) \quad \text { in } \quad \mathscr{D}^{\prime}(\Omega \backslash \bar{D})
$$

and an evolutionary quasi-variational inequality over D,

$$
\begin{aligned}
\|\nabla u(t)\|_{L^{\infty}(D)} & \leq 1, \\
\int_{D}\left(f(x, t)-\partial_{t} u(x, t)\right)(z(x)-u(x, t)) d x & \leq 0 \quad \text { for all } z \in K_{D}(u(t)),
\end{aligned}
$$

where $K_{D}(w)$ is given for each $w \in W_{0}^{1, q^{-}}(\Omega)$ by

$$
K_{D}(w):=\left\{z \in W^{1, \infty}(D) ; z-w \in W_{0}^{1, q^{-}}(D) \text { and }\|\nabla z\|_{L^{\infty}(D)} \leq 1\right\} .
$$

In addition, if

$$
\int_{\Omega} \frac{1}{p_{n}(x)}\left|\nabla u_{0, n}(x)\right|^{p_{n}(x)} d x \rightarrow \int_{\Omega \backslash \bar{D}} \frac{1}{q(x)}\left|\nabla u_{0}(x)\right|^{q(x)} d x,
$$

then (5.11) holds and

$$
u_{n} \rightarrow u \quad \text { strongly in } L^{r}\left(0, T ; X^{q(\cdot)}(\Omega \backslash \bar{D})\right) \quad \text { for each } r \in[1, \infty) \text {. }
$$

Remark 6.2. $\quad$ 1. Roughly speaking, the constraint set of the evolutionary quasi-variational inequality requires all test functions $z$ in (6.8) to coincide with $u(\cdot, t)$ on the boundary $\partial D$ at each time $t$.

2. From the assumptions, $q(\cdot)<\infty$ in $\Omega \backslash \bar{D}$ and $q_{n}^{-} \rightarrow \infty$, the exponents $p_{n}(\cdot)$ must be discontinuous on $\partial D$ for $n \in \mathbb{N}$ large enough. So we need work in the framework of discontinuous exponents (see Sect. 3).

To prove this theorem, we first show the Mosco convergence of $\varphi_{n}$. 
Proposition 6.3. (Mosco convergence) Suppose that (6.3) holds. Then $\varphi_{n}$ Mosco-converges on $L^{2}(\Omega)$ to the functional $\varphi_{D}: L^{2}(\Omega) \rightarrow[0, \infty]$ given by

$$
\varphi_{D}(w):=\left\{\begin{array}{cc}
\int_{\Omega \backslash \bar{D}} \frac{1}{q(x)}|\nabla w(x)|^{q(x)} d x & \text { if } w \in W_{0}^{1, q^{-}}(\Omega), w \in X^{q(\cdot)}(\Omega \backslash \bar{D}) \\
\infty & \text { and }\|\nabla w\|_{L^{\infty}(D)} \leq 1, \\
\text { otherwise }
\end{array}\right.
$$

as $n \rightarrow \infty$.

Proof. Let $u \in D\left(\varphi_{D}\right)$ be fixed and set $u_{n}=u$ for all $n \in \mathbb{N}$. Then $u \in L^{2}(\Omega)$, and it also holds that $\partial u / \partial x_{i} \in L^{p_{n}(\cdot)}(\Omega)$ for $i=1,2, \ldots, N$. Moreover, since $p_{n}^{-}=q^{-}$for any $n \in \mathbb{N}$ large enough, we have $W_{0}^{1, q^{-}}(\Omega)=W_{0}^{1, p_{n}^{-}}(\Omega)$. Hence $u \in X_{0}^{p_{n}(\cdot)}(\Omega)=D\left(\varphi_{n}\right)$ for sufficiently large $n \in \mathbb{N}$. We observe that

$$
\begin{aligned}
\varphi_{n}\left(u_{n}\right) & =\int_{D} \frac{1}{q_{n}(x)}|\nabla u(x)|^{q_{n}(x)} d x+\int_{\Omega \backslash \bar{D}} \frac{1}{q(x)}|\nabla u(x)|^{q(x)} d x \\
& \rightarrow \int_{\Omega \backslash \bar{D}} \frac{1}{q(x)}|\nabla u(x)|^{q(x)} d x=\varphi_{D}(u),
\end{aligned}
$$

since it follows from (6.3) that

$$
\int_{D} \frac{1}{q_{n}(x)}|\nabla u(x)|^{q_{n}(x)} d x \leq \frac{|D|}{q_{n}^{-}} \rightarrow 0 .
$$

Thus (i) of Definition 5.1 holds for $\varphi_{n}$.

We next prove (ii) of Definition 5.1. Let $u_{n} \in D\left(\varphi_{n}\right)$ be such that $u_{n} \rightarrow u$ weakly in $L^{2}(\Omega)$. It is sufficient to treat the case that

$$
\liminf _{n \rightarrow \infty} \varphi_{n}\left(u_{n}\right)<\infty .
$$

Then, up to a subsequence, we have $\varphi_{n}\left(u_{n}\right) \leq C$, which brings us two observations. The first one reads,

$$
\int_{\Omega \backslash \bar{D}} \frac{1}{q(x)}\left|\nabla u_{n}(x)\right|^{q(x)} d x \leq C,
$$

which implies, up to a subsequence, $\nabla u_{n} \rightarrow \nabla u$ weakly in $\left(L^{q(\cdot)}(\Omega \backslash \bar{D})\right)^{N}$. Hence $u \in X^{q(\cdot)}(\Omega \backslash \bar{D})$. The second one is the following:

$$
\int_{D} \frac{1}{q_{n}(x)}\left|\nabla u_{n}(x)\right|^{q_{n}(x)} d x \leq C .
$$

Repeating the same argument as in the proof of Proposition 5.5, since $q_{n}^{-}-1>$ $q^{-}$for sufficiently large $n \in \mathbb{N}$, one can also derive

$$
\left\|\nabla u_{n}\right\|_{L^{q^{-}}(D)} \leq C
$$

which together with (6.11) yields the boundedness of $\left(\nabla u_{n}\right)$ in $\left(L^{q^{-}}(\Omega)\right)^{N}$. By Poincaré's inequality for usual Sobolev spaces, $\left(u_{n}\right)$ is bounded in $W_{0}^{1, q^{-}}(\Omega)$, and therefore, up to a subsequence, $u_{n} \rightarrow u$ weakly in $W_{0}^{1, q^{-}}(\Omega)$ and $u \in$ $W_{0}^{1, q^{-}}(\Omega)$. Moreover, it can be also proved by (6.12) as in Sect. 5 that 


$$
\|\nabla u\|_{L^{\infty}(D)} \leq 1 .
$$

Thus combining these facts, we deduce that $u \in D\left(\varphi_{D}\right)$.

Since the functional $u \mapsto \int_{\Omega \backslash \bar{D}}(1 / q(x))|\nabla u(x)|^{q(x)} d x$ is weakly lower semicontinuous in $L^{2}(\Omega)$, it follows that

$$
\begin{aligned}
\varphi_{D}(u) & =\int_{\Omega \backslash \bar{D}} \frac{1}{q(x)}|\nabla u(x)|^{q(x)} d x \\
& \leq \liminf _{n \rightarrow \infty} \int_{\Omega \backslash \bar{D}} \frac{1}{q(x)}\left|\nabla u_{n}(x)\right|^{q(x)} d x \\
& \leq \liminf _{n \rightarrow \infty} \varphi_{n}\left(u_{n}\right) .
\end{aligned}
$$

Consequently, (ii) of Definition 5.1 follows.

We are now ready to prove Theorem 6.1.

Proof of Theorem 6.1. One can prove the convergence of $u_{n}$ and observe that the limit $u$ uniquely solves

$$
\frac{d u}{d t}(t)+\partial \varphi_{D}(u(t)) \ni f(t) \text { in } H=L^{2}(\Omega), \quad u(0)=u_{0}
$$

by applying Proposition 5.2 with $\phi_{n}=\varphi_{n}$ and $\phi=\varphi_{D}$. Hence the main task of our proof is to obtain a representation of (6.13). We first claim that

$$
\xi=-\Delta_{q(\cdot)} w \text { in } \mathscr{D}^{\prime}(\Omega \backslash \bar{D}) \quad \text { if } \quad \xi \in \partial \varphi_{D}(w) .
$$

By the definition of subdifferentials,

$$
\varphi_{D}(v)-\varphi_{D}(w) \geq \int_{\Omega} \xi(x)(v(x)-w(x)) d x \quad \text { for all } v \in D\left(\varphi_{D}\right) .
$$

In particular, put

$$
v(x)= \begin{cases}w(x) & \text { in } D, \\ w(x)+h e(x) & \text { in } \Omega \backslash \bar{D}\end{cases}
$$

with arbitrary $h \in \mathbb{R}$ and $e \in C_{0}^{\infty}(\Omega \backslash \bar{D})$. Then $v$ belongs to $D\left(\varphi_{D}\right)$ and we observe that

$$
\begin{aligned}
& \int_{\Omega \backslash \bar{D}} \frac{1}{q(x)}|\nabla w(x)+h \nabla e(x)|^{q(x)} d x-\int_{\Omega \backslash \bar{D}} \frac{1}{q(x)}|\nabla w(x)|^{q(x)} d x \\
& \quad \geq h \int_{\Omega \backslash \bar{D}} \xi(x) e(x) d x .
\end{aligned}
$$

Thus we derive

$$
\int_{\Omega \backslash \bar{D}}|\nabla w(x)|^{q(x)-2} \nabla w(x) \cdot \nabla e(x) d x=\int_{\Omega \backslash \bar{D}} \xi(x) e(x) d x
$$

for all $e \in C_{0}^{\infty}(\Omega \backslash \bar{D})$, and therefore, $\xi=-\Delta_{q(\cdot)} w$ in $\mathscr{D}^{\prime}(\Omega \backslash \bar{D})$.

We next claim that

$$
\int_{D} \xi(x)(z(x)-w(x)) d x \leq 0 \text { for all } z \in K_{D}(w) \quad \text { if } \xi \in \partial \varphi_{D}(w) \text {. }
$$


Indeed, let $z \in K_{D}(w)$ and substitute the following to (6.15):

$$
v(x)=\left\{\begin{array}{l}
z(x) \text { in } D, \\
w(x) \text { in } \Omega \backslash \bar{D} .
\end{array}\right.
$$

Here we remark that $v \in D\left(\varphi_{D}\right)$, because obviously $v \in X^{q(\cdot)}(\Omega \backslash \bar{D})$ and $\|\nabla v\|_{L^{\infty}(D)} \leq 1$; the zero extension $\overline{z-w}$ of $z-w \in W_{0}^{1, q^{-}}(D)$ into $\Omega$ belongs to $W_{0}^{1, q^{-}}(\Omega)$ and hence $v=\overline{z-w}+w \in W_{0}^{1, q^{-}}(\Omega)$. Then (6.16) follows. Consequently, by (6.14) and (6.16), the Cauchy problem (6.13) is rewritten to (6.4)-(6.8).

We finally prove $(6.10)$ under $(6.9)$. Since $\varphi_{n}\left(u_{n}(\cdot)\right) \rightarrow \varphi_{D}(u(\cdot))$ uniformly on $[0, T]$ by Proposition 5.2, we observe

$$
\begin{aligned}
\int_{\Omega \backslash \bar{D}} \frac{1}{q(x)}|\nabla u(x, t)|^{q(x)} d x & =\varphi_{D}(u(t)) \\
& =\lim _{n \rightarrow \infty} \varphi_{n}\left(u_{n}(t)\right) \\
& \geq \limsup _{n \rightarrow \infty} \int_{\Omega \backslash \bar{D}} \frac{1}{q(x)}\left|\nabla u_{n}(x, t)\right|^{q(x)} d x .
\end{aligned}
$$

Recall $\nabla u_{n} \rightarrow \nabla u$ weakly in $\left(L^{q(\cdot)}(\Omega \backslash \bar{D})\right)^{N}$ and note that

$$
\liminf _{n \rightarrow \infty} \int_{\Omega \backslash \bar{D}} \frac{1}{q(x)}\left|\nabla u_{n}(x, t)\right|^{q(x)} d x \geq \int_{\Omega \backslash \bar{D}} \frac{1}{q(x)}|\nabla u(x, t)|^{q(x)} d x .
$$

Hence as in the proof of Theorem 3.4, it follows that $u_{n}(t) \rightarrow u(t)$ strongly in $X^{q(\cdot)}(\Omega \backslash \bar{D})$ for every $t \in[0, T]$. Since $\varphi_{n}\left(u_{n}(t)\right)$ is uniformly bounded over $[0, T]$ for all $n \in \mathbb{N}$, by Lebesgue's dominated convergence theorem, $u_{n}$ converges to $u$ strongly in $L^{r}\left(0, T ; X^{q(\cdot)}(\Omega \backslash \bar{D})\right)$ for any $r \in[1, \infty)$.

\section{Acknowledgments}

The authors are grateful to Professor Mokhtar Kirane for fruitful discussion at Dresden in 2010.

\section{References}

[1] Acerbi, E., Mingione, G.: Regularity results for a class of functionals with nonstandard growth. Arch. Ration. Mech. Anal. 156, 121-140 (2001)

[2] Acerbi, E., Mingione, G.: Regularity results for stationary electro-rheological fluids. Arch. Ration. Mech. Anal. 164, 213-259 (2002)

[3] Acerbi, E., Mingione, G., Seregin, G.A.: Regularity results for parabolic systems related to a class of non-Newtonian fluids. Ann. Inst. H. Poincaré Anal. Non Linéaire 21, 25-60 (2004)

[4] Akagi, G.: Convergence of functionals and its applications to parabolic equations. Abstr. Appl. Anal. 2004, 907-933 (2004) 
[5] Akagi, G., Matsuura, K.: Well-posedness and large-time behaviors of solutions for a parabolic equation involving $p(x)$-Laplacian. "The Eighth International Conference on Dynamical Systems and Differential Equations," a supplement volume of Discrete and Continuous Dynamical Systems, pp.22-31 (2011)

[6] Akagi, G., Ôtani, M.: Time-dependent constraint problems arising from macroscopic critical-state models for type-II superconductivity and their approximations. Adv. Math. Sci. Appl. 14, 683-712 (2004)

[7] Antontsev, S., Shmarev, S.: Extinction of solutions of parabolic equations with variable anisotropic nonlinearities. Tr. Mat. Inst. Steklova 261. Differ. Uravn. i Din. Sist., pp. 16-25 (2008)

[8] Antontsev, S., Shmarev, S.: Anisotropic parabolic equations with variable nonlinearity. Publ. Mat. 53, 355-399 (2009)

[9] Antontsev, S., Shmarev, S.: Vanishing solutions of anisotropic parabolic equations with variable nonlinearity. J. Math. Anal. Appl. 361, 371-391 (2010)

[10] Antontsev, S., Shmarev, S.: Blow-up of solutions to parabolic equations with nonstandard growth conditions. J. Comput. Appl. Math. 234, 2633-2645 (2010)

[11] Aronsson, G., Evans, L.C., Wu, Y.: Fast/slow diffusion and growing sandpiles. J. Differ. Equ. 131, 304-335 (1996)

[12] Attouch, H.: Variational Convergence for Functions and Operators. Applicable Mathematics Series. Pitman (Advanced Publishing Program), Boston (1984)

[13] Barrett, J.W., Prigozhin, L.: Bean's critical-state model as the $p \rightarrow \infty$ limit of an evolutionary $p$-Laplacian equation. Nonlinear Anal. 42, 977-993 (2000)

[14] Berryman, J.G., Holland, C.J.: Stability of the separable solution for fast diffusion. Arch. Ration. Mech. Anal. 74, 379-388 (1980)

[15] Bendahmane, M., Wittbold, P., Zimmermann, A.: Renormalized solutions for a nonlinear parabolic equation with variable exponents and $L^{1}$-data. J. Differ. Equ. 249, 1483-1515 (2010)

[16] Brézis, H.: Opérateurs Maximaux Monotones et Semi-Groupes de Contractions dans les Espaces de Hilbert. Math Studies, vol.5. North-Holland, Amsterdam (1973)

[17] Chen, Y., Levine, S., Rao, M.: Variable exponent, linear growth functionals in image restoration. SIAM J. Appl. Math. 66, 1383-1406 (2006)

[18] DiBenedetto, E.: Degenerate Parabolic Equations. Universitext, SpringerVerlag, New York (1993)

[19] Diening, L., Ettwein, F., Růžička, M.: $C^{1, \alpha}$-regularity for electrorheological fluids in two dimensions. Nonlinear Differ. Equ. Appl. 14, 207-217 (2007) 
[20] Diening, L., Harjulehto, P., Hästö, P., Růžička, M.: Lebesgue and Sobolev Spaces with Variable Exponents. Lecture Notes in Mathematics, vol. 2017., Springer, Heidelberg (2011)

[21] Ettwein, F., Růžička, M.: Existence of local strong solutions for motions of electrorheological fluids in three dimensions. Comput. Math. Appl. 53, 595-604 (2007)

[22] Edmunds, D.E., Rákosník, J.: Sobolev embeddings with variable exponent. Studia Math. 143, 267-293 (2000)

[23] Edmunds, D.E., Rákosník, J.: Sobolev embeddings with variable exponent II. Math. Nachr. 246/247, 53-67 (2002)

[24] Fan, X., Shen, J., Zhao, D.: Sobolev embedding theorems for spaces $W^{k, p(x)}(\Omega)$. J. Math. Anal. Appl. 262, 749-760 (2001)

[25] Fan, X., Zhao, D.: On the spaces $L^{p(x)}(\Omega)$ and $W^{m, p(x)}(\Omega)$. J. Math. Anal. Appl. 263, 424-446 (2001)

[26] Fan, X., Zhao, Y., Zhao, D.: Compact imbedding theorems with symmetry of Strauss-Lions type for the space $W^{1, p(x)}(\Omega)$. J. Math. Anal. Appl. 255, 333-348 (2001)

[27] Fu, Y., Pan, N.: Existence of solutions for nonlinear parabolic problem with $p(x)$-growth. J. Math. Anal. Appl. 362, 313-326 (2010)

[28] Harjulehto, P., Hästö, P., Lê, Ú.-V., Nuortio, M.: Overview of differential equations with non-standard growth. Nonlinear Anal. 72, 4551-4574 (2010)

[29] Kováčik, O., Rákosník, J.: On spaces $L^{p(x)}$ and $W^{k, p(x)}$. Czechoslovak Math. J. 41, 592-618 (1991)

[30] Manfredi, J.J., Rossi, J.D., Urbano, J.M.: $p(x)$-harmonic functions with unbounded exponent in a subdomain. Ann. Inst. H. Poincaré Anal. Non Linéaire 26, 2581-2595 (2009)

[31] Manfredi, J.J., Rossi, J.D., Urbano, J.M.: Limits as $p(x) \rightarrow \infty$ of $p(x)$-harmonic functions. Nonlinear Anal. 72, 309-315 (2010)

[32] Musielak, J.: Orlicz Spaces and Modular Spaces. Lecture Notes in Mathematics, vol. 1034. Springer, Berlin (1983)

[33] Qi, Y.-W.: The degeneracy of a fast-diffusion equation and stability. SIAM J. Math. Anal. 27, 476-485 (1996)

[34] Růžička, M.: Electrorheological Fluids: Modeling and Mathematical Theory. Lecture Notes in Mathematics, vol. 1748 Springer, Berlin (2000)

[35] Samko, S.: On a progress in the theory of Lebesgue spaces with variable exponent: maximal and singular operators. Integr. Transforms Spec. Funct. 16, 461-482 (2005) 
[36] Sanchón, M., Urbano, J.M.: Entropy solutions for the $p(x)$-Laplace equation. Trans. Am. Math. Soc. 361, 6387-6405 (2009)

[37] Urbano, J.M., Vorotnikov, D.: On the well-posedness of a two-phase minimization problem. J. Math. Anal. Appl. 378, 159-168 (2011)

[38] Zhang, C., Zhou, S.: Renormalized and entropy solutions for nonlinear parabolic equations with variable exponents and $L^{1}$ data. J. Differ. Equ. 248, 13761400 (2010)

[39] Zhikov, V.V.: On the technique for passing to the limit in nonlinear elliptic equations. Funct. Anal. Appl. 43, 96-112 (2009)

Goro Akagi

Graduate School of System Informatics

Kobe University

1-1 Rokkodai-cho

Nada-ku

Kobe 657-8501

Japan

e-mail: akagi@port.kobe-u.ac.jp

Kei Matsuura

Research Institute for Science and Engineering

Waseda University

3-4-1 Okubo

Shinjuku-ku

Tokyo 169-8555

Japan

e-mail: kei@kurenai.waseda.jp

Received: 1 December 2011.

Accepted: 17 January 2012. 